\title{
Riqueza, distribución y conservación de los topos y las musarañas (Mammalia, Eulipotyphla) de México
}

\section{Lázaro Guevara ${ }^{1,2^{*}}$, Fernando A. Cervantes ${ }^{2}$ y Víctor Sánchez-Cordero²}

'Posgrado en Ciencias Biológicas, Universidad Nacional Autónoma de México. Av. Ciudad Universitaria 3000, Coyoacán 04360, Distrito Federal, México. E-mail: Ilg@st.ib.unam.mx (LG),

${ }^{2}$ Departamento de Zoología, Instituto de Biología, Universidad Nacional Autónoma de México. Av. Ciudad Universitaria 3000, Apartado Postal 70-153, Coyoacán 04360, Distrito Federal, México. E-mail: fac@ib.unam.mx (FAC). victor@ib.unam.mx (VSC).

${ }^{*}$ Corresponding author

Introduction: Moles (Talpidae) and shrews (Soricidae) are the only representatives of the order Eulipotyphla in Mexico and they account for $7 \%$ of mammals in the country. Despite their richness, even basic aspects such as their species-level taxonomic knowledge and geographical distribution are still uncertain. The scarcity of such information implies that the biology and conservation status of eulipotyphlans are also unclear or unevaluated, which involves a serious impediment to the design of management strategies for a group that tends to be susceptible to climate change and impacts of anthropogenic habitat transformation. Here, we performed a comprehensive assessment of the current state of knowledge and threats to the survival of Mexican moles and shrews using information from natural history collections and spatial environmental data.

Methods: We reviewed the available information in biological collections, databases, and literature records of Mexican eulipotyphlans, and evaluated the bias road in the collection of specimens. The current distribution was estimated for nearly all moles and shrews recorded in Mexico, using ecological niche modeling and retaining the remnant vegetation areas. Finally, we calculated the extent of distribution for each species within protected areas and within the most threatened ecosystems in Mexico to identify the most vulnerable taxa.

Results: The eulipotyphlan diversity of Mexico is represented by three species of moles and 36 of shrews. Of all these, 26 species (67\%) are endemic to the country and 27 (69\%) are listed in a risk category by Mexican government or global assessments. Eleven taxa are known only from no more ten specimens or from very few collecting sites. The shrew Sorex stizodon has not been recorded for more than a century. Current distributions of twelve species were not estimated because they are represented by just a few locality records $(<5)$. The region that could contain most taxonomic richness is the highlands of central and southern Mexico. The species with the highest percentage of transformed habitat are the mole Scalopus latimanus and the shrews Cryptotis merriami, C. mexicanus, C. obscurus, and Sorex ornatus. Based on the current distribution, the number of records, the current protection within AP and /or potential threats, Cryptotis griseoventris and the recently described C. lacandonensis should be protected by the Mexican government.

Discussion and Conclusions: This paper provides the first detailed documentation of available information on the taxonomy, nomenclature, current distribution, and threats of moles and shrews in Mexico. Information from natural history collections corroborates the sparse and biased knowledge about the distribution of eulipotyphlans (RamírezPulido et al. 2005; Carraway 2007). Our spatial analyses provide evidence that several species may be more endangered than suggested by global approaches (IUCN) and Mexican government legislation. Several species of moles and shrews may be represented sparsely in collections because of insufficient collecting. Our niche models projected onto a map to identify the distribution should be used in directing field survey efforts and scientific collecting in order to increase the information regarding the current population status of moles and shrews.

Key words: bias road, current distribution, field surveys, Insectivora, natural history collections, small mammals. 


\section{Introducción}

El orden Eulipotyphla es un grupo monofilético que incluye a los erizos del Viejo Mundo (Erinaceidae), a los solenodontes (Solenodontidae) de las Antillas Mayores, a las musarañas (Soricidae) y a los topos (Talpidae; Stanhope et al. 1998; Douady et al. 2002). Anteriormente se agrupaban en el orden Insectivora junto a los topos dorados del sur de África (Chrysochloridae) y a los tenrecs originarios de Madagascar (Tenrecidae); actualmente, estas dos últimas familias pertenecen a un clado africano denominado Afrotheria (Stanhope et al. 1998). Otros esquemas alternativos de clasificación han agrupado a todas estas familias en diversos órdenes, como Soricomorpha, Erinaceomorpha y Lipotyphla (Simpson 1945; Hutterer 2005; Ramírez-Pulido et al. 2008; Asher y Helgen 2010; Wilson y Redder 2011). Lo cual refleja que la taxonomía y sistemática de estos mamíferos pequeños han sido un reto constante dentro del estudio evolutivo de los mamíferos (Simpson 1945; Stanhope et al. 1998; Douady et al. 2002; Douady y Douzery 2003; Nikaido et al. 2003; Symonds 2005).

El linaje que dio origen al orden Eulipotyphla es tan antiguo en la historia de los mamíferos que incluso precede a la extinción de los dinosaurios, hace cerca de 66 millones de años (Douady y Douzery 2003; Roca et al. 2004). El tiempo trascurrido desde su origen ha producido un grupo con más de 450 especies que se distribuyen en casi todo el planeta y con una gran diversidad de formas y estrategias de vida (Symonds 2005). En México, los eulipotiflos están representados por los topos y las musarañas (Maldonado 1999; Carraway 2007), con más de 35 especies que, en general, se caracterizan por su tamaño relativamente pequeño y por alimentarse principalmente de insectos y artrópodos. La mayoría de los topos son fosoriales, es decir, están adaptados a una vida debajo de la tierra, tienen forma de torpedo, poseen ojos y orejas diminutas y los sentidos olfativo, auditivo y táctil están bien desarrollados (Symonds 2005; Merritt 2010). Los topos mexicanos habitan marginalmente en el extremo norte del país (Maldonado 1999). Por su parte, las musarañas se encuentran entre los mamíferos terrestres de menor tamaño en el planeta, poseen ojos diminutos, cabeza alargada y hocico particularmente puntiagudo y, debido a su apetito voraz, se mantienen activas tanto en el día como en la noche (Churchfield 2002). Las musarañas están distribuidas en casi todo el territorio mexicano (Carraway 2007). Los eulipotiflos integran uno de los componentes mastozoológicos de la biodiversidad en México más importantes en cuanto a su número de especies, apenas por detrás de los roedores, murciélagos, carnívoros y artiodáctilos (incluyendo cetáceos, Asher y Helgen 2010).

El común denominador para ambas familias es que son poco estudiadas y se encuentran entre los mamíferos menos conocidos del país (Carraway 2007; Cervantes et al. 2008). Quizá la dificultad que implica, tanto su captura como la identificación taxonómica de los taxones, son factores que han limitado su estudio (Ramírez-Pulido et al. 2005; Carraway 2007; Woodman et al. 2012). Como consecuencia, el número de especies reconocidas podría ser incierto, sus distribuciones geográficas parcialmente conocidas y su estado de conservación poco evaluado (Cervantes et al. 2008; Guevara et al. 2014a; Guevara et al. 2014b). El notable vacío de información en el conocimiento de su riqueza taxonómica y distribución geográfica no ha permitido realizar una adecuada valoración del estado de conservación y las amenazas que enfrentan en México. Los topos y las musarañas son potencialmente vulnerables a los efectos de los cambios asociados con la destrucción del hábitat inducida por el humano y por el cambio climático (Schloss et al. 2012; Woodman et al. 2012).

Los estudios recientes sobre eulipotiflos mexicanos incluyen la revalidación y descripción de especies (Yates y Salazar-Bravo 2005; Carraway 2007; Guevara et al. 2014b) y estudios sobre taxonomía y sistemática filogenética (Ramírez-Pulido et al. 2004, 2005; Esteva et al. 2010; Woodman 2010; Guevara y Cervantes 2014). Estos esfuerzos han marcado el inicio de la utilización integral 
de los datos obtenidos en acervos biológicos con información geográfica que están siendo manejados para incrementar nuestro conocimiento sobre taxonomía y aspectos ecológicos y evolutivos, que repercuten en la planeación de estrategias de conservación (Graham et al. 2004; Sánchez-Cordero et al. 2001, 2005).

En este sentido, en las últimas décadas se ha desarrollado el modelado del nicho ecológico (MNE), una herramienta que brinda la opción de combinar la información geográfica relacionada a los ejemplares de museo con variables bioclimáticas que caractericen los requerimientos ambientales de las especies para mantener poblaciones viables (Soberón y Peterson 2005). De esta forma, el MNE permite identificar regiones en el espacio geográfico donde una especie no ha sido registrada, pero es probable que ocurra (Sánchez-Cordero et al. 2001, 2005). Por esto, los MNE se han aplicado ampliamente en muchos estudios relacionados con la delimitación de las distribuciones de especies y la planeación de estrategias de conservación (Peterson et al. 2011), incluso para taxones con un número reducido de registros (Pearson et al. 2007; Jackson y Robertson 2011). Por lo anterior, los objetivos de la presente contribución son explorar y dar a conocer la información disponible en colecciones biológicas y bases de datos en torno al orden Eulipotyphla en México, integrar los cambios nomenclaturales y de clasificación taxonómica recientes $y$, finalmente, modelar el nicho ecológico de las especies para estimar sus distribuciones actuales e identificar a los linajes más vulnerables en el país.

\section{Material y Métodos}

Información taxonómica y geográfica. La actualización taxonómica y nomenclatural se basó en las evaluaciones del estado taxonómico, descripción de taxones nuevos y en propuestas nomenclaturales recientes (Matson y McCarthy 2005; Yates y Salazar-Bravo 2005; Carraway 2007; Esteva et al. 2010; Woodman 2012; Woodman et al. 2012; Guevara et al. 2014a, 2014b; RamírezPulido et al. 2014). Para la obtención de los datos geográficos se solicitaron los registros contenidos en el Sistema Nacional de Información sobre Biodiversidad (SNIB) de la Comisión Nacional para el Conocimiento y Uso de la Biodiversidad (CONABIO) y se consultaron las bases de datos del Mammal Networked Information System (MaNIS; http://www.manisnet.org) y el Global Biodiversity Information Facility (GBIF; http://www.gbif.org) para un registro inicial de 15,732 ejemplares. Una vez teniendo las localidades en grados decimales $(n=7,778)$, se realizó la validación geográfica de los registros de cada especie sobreponiendo la capa de localidades de cada una de las especies sobre el polígono estimado por la Unión Internacional para la Conservación de la Naturaleza (IUCN 2013). A la vez, se consultaron las siguientes colecciones biológicas para validar la información taxonómica y geográfica: Colección Zoológica Regional (Mammalia) del Instituto de Historia Natural y Ecología, Tuxtla Gutiérrez, Chiapas (CZRMA); Colección Nacional de Mamíferos, Distrito Federal (CNMA); Colección Mastozoológica El Colegio de la Frontera Sur, San Cristóbal de Las Casas, Chiapas (ECO-SC-M); The University of Kansas, Natural History Museum, Lawrence (KU); Museo de Zoología "Alfonso L. Herrera", Distrito Federal (MZFC); Universidad Autónoma Metropolitana Unidad Iztapalapa, Distrito Federal (UAMI); Universidad Autónoma de Yucatán, Mérida, Yucatán (UADY); Colección Osteológica del Laboratorio de Arqueozoología "M. en C. Ticul Álvarez Solórzano", Distrito Federal (INAH); Colección de Mamíferos del Centro de Educación Ambiental e Investigación Sierra de Huautla, UAEM, Cuernavaca, Morelos (CIByC); Colección Regional Mastozoológica del CIIDIR-Oaxaca, Oaxaca de Juárez (OAXMA) y el National Museum of Natural History, Smithsonian Institution,Washington (USNM). Se puso especial atención en el número de ejemplares depositados en colecciones biológicas por cada especie como indicativo del conocimiento de las especies. 
Modelado de nicho ecológico. La estimación del nicho ecológico se realizó con el método de máxima entropía en el programa Maxent v. 3.3 (Phillips et al. 2006), ya que proporciona información útil y establece una aproximación de la distribución de múltiples taxones (Sánchez-Cordero et al. 2005). Al estimar la distribución de las especies se requiere definir el área de estudio en la cual se calibra el modelo (ver Peterson et al. 2011) y debe hacerse en función del área accesible que tiene la especie para expandir su distribución (región ' $M$ ' en diagrama BAM de Soberón y Peterson 2005). La selección de las áreas de estudio por especie se realizó al intersectar los registros validados con la regionalización biogeográfica de las Ecorregiones Terrestres del Mundo a Nivel III (Commission for Environmental Cooperation; http://www.cec. org), más un buffer adicional de 40 kilómetros para permitir la inclusión de áreas con registro fósil, en los casos en los que existen (Choate 1970; Carraway 2007). Ante la falta de un mayor conocimiento biogeográfico de las especies, consideramos que esta selección de áreas de calibración es un supuesto explícito que puede ser puesto a prueba conforme se obtenga mayor evidencia.

Las coberturas bioclimáticas seleccionadas como predictores de la distribución fueron 19 variables bioclimáticas (http://www. worldclim.org): temperatura promedio anual (bio01), oscilación diurna de la temperatura (bio02), isotermalidad (bio03), estacionalidad de la temperatura (bio04), temperatura máxima promedio del periodo más cálido (bio05), temperatura mínima promedio del periodo más frío (bio06), oscilación anual de la temperatura (bio07), temperatura promedio del trimestre más Iluvioso (bio08), temperatura promedio del trimestre más seco (bio09), temperatura promedio del trimestre más cálido (bio10), temperatura promedio del trimestre más frío (bio11), precipitación anual (bio12), precipitación del periodo más lluvioso (bio13), precipitación del periodo más seco (bio14), estacionalidad de la precipitación (bio15), precipitación del trimestre más lluvioso (bio16), precipitación del trimestre más seco (bio17), precipitación del trimestre más cálido (18), precipitación del trimestre más frío (bio19) y la variable topográfica que indica la pendiente del terreno (pen; http:// edc.usgs. gov/products/ elevation/gtopo30/hydro), cada una con resolución de 30 segundos $\left(\approx 1 \mathrm{~km}^{2}\right)$. Todas las capas fueron recortadas para su análisis de acuerdo al área seleccionada de cada especie. Con la intención de evitar el problema de la multicolinealidad entre variables, se usó el coeficiente de correlación de Pearson para eliminar a las variables bioclimáticas correlacionadas para cada especie en el programa ENMTools (Warren et al. 2010; Tablas 1 y 2).

No se generaron modelos de distribución para las especies en las que los registros son insuficientes $(n \leq 4)$. Para el caso de las especies con $\geq 5 y \leq 25$ registros utilizamos el método de Jackknife (Pearson et al. 2007; Tabla 1). En este enfoque, el modelo se calibra usando $n-1$ registros y se evalúa la capacidad de predecir el registro faltante en la etapa de calibración, repitiendo el proceso hasta que todos los registros se han utilizado para la evaluación. Este método requiere la aplicación de un umbral (Pearson et al. 2007; Jackson y Robertson 2011). Se eligió el valor más bajo predicho que está asociado con cualquiera de los registros de presencia observados (umbral de presencia más bajo, Pearson et al. 2007; Minimum training presence en Maxent). Este umbral puede interpretarse como el reconocimiento de píxeles que son al menos tan adecuados para la especie, como aquellos en los que se ha confirmado su presencia. Dicho umbral se eligió debido al control estricto sobre la presencia para estas especies y a que la intención es detectar regiones que potencialmente podrían albergar poblaciones aún no descubiertas (Peterson et al. 2011). Se usó el 
software desarrollado por Pearson et al. (2007) para calcular el porcentaje de registros predichos correctamente y el $P$-valor para evaluar la significancia de las predicciones.

Para el caso de las especies con $\geq 26$ registros (Tabla 2), se utilizó el método de validación cruzada ( $k$-fold) para evaluar la consistencia de los modelos por cada especie usando diferentes subconjuntos de datos para la calibración, permitiendo un máximo de 50 réplicas. Debido a la incertidumbre en el límite taxonómico entre las musarañas Cryptotis mexicanus y C. obscurus (Choate 1970; Guevara y Cervantes 2014), se utilizaron los registros por arriba de los $20^{\circ} 30^{\prime}$ para C. obscurus y por debajo de los $20^{\circ}$ para C. mexicanus (ver Choate 1970). La validación independiente del umbral de estos modelos se determinó mediante el área bajo la curva (AUC) del análisis Receiver Operating Characteristic (ROC). Se incluye la diferencia entre los datos de calibración y los de evaluación $\left(A \cup C_{\mathrm{di}}\right)$. Entre menor sea la diferencia entre los datos de calibración y evaluación, menor es el riesgo de que los modelos estén sobre ajustados, es decir, que sean específicos a los datos de calibración (Warren y Seifter 2011). Los valores de $A U C_{e v}$ (de evaluación) y $A U C_{d i}$ son apropiados para las comparaciones de modelos producidos para la misma especie, en el mismo sitio de estudio o área de calibración y bajo diferentes parámetros (Anderson y González Jr. 2011). Para la evaluación dependiente del umbral, se permitió error de comisión al incluir el 90 \% de los datos de calibración (10 percentile training presence en Maxent).

Sesgo de muestreo. Maxent compara los valores ambientales en los registros de presencia de las especies con puntos generados a través del área de estudio conocidos como background, los cuales son generados bajo el supuesto de que toda el área ha sido muestreada sin sesgo (Phillips et al. 2006). Aquí se evaluó si existe un sesgo en el muestreo dirigido hacia las carreteras, ya que este sesgo geográfico podría estar acompañado de sesgo ambiental que afectaría a los modelos (Phillips et al. 2009). Debido a la escasez de registros de la familia Talpidae para México, este análisis solo se realizó para la familia Soricidae. Para ello, se utilizó la ruta de caminos en formato shapefile disponible a través del Digital Chart of the World (http://www.princeton. edu). Se importaron los puntos de colecta para la familia en formato shapefile (datos observados; 651 localidades únicas) y calculamos la distancia de cada punto al camino más cercano. Después, se generó el mismo número de puntos al azar y se calcularon las distancias a los caminos con el fin de evaluar si ambas distribuciones de puntos son diferentes mediante una prueba no-paramétrica para dos muestras independientes (prueba de Mann-Whitney) en el paquete estadístico Statistica (STATSOFT INC. 2005).

Con base en este cálculo, se creó un buffer hacia los caminos igual a la media más 1 desviación estándar del valor observado en los registros y se generó un archivo en formato ASCII para poder ingresarlo al programa Maxent. Al usar este archivo de sesgo, se espera que los datos de background posean el mismo sesgo que los datos de presencia (Phillips et al. 2009). Con fines de comparación, todos los MNE se realizaron con y sin información sobre el sesgo en el muestreo. Los mapas presentados por especie (Apéndice 1) se seleccionaron de acuerdo a los resultados de la evaluación del modelo y a la inspección visual en el espacio geográfico.

Distribución actual y análisis de amenazas. Se convirtió la distribución estimada a la distribución actual al contabilizar y excluir zonas transformadas por el hombre que reducen la posibilidad de encontrar poblaciones viables de las especies (zonas urbanas, desprovisto de vegetación, asentamientos humanos, sin vegetación aparente; Serie V; INEGI 2014). Con la finalidad de conocer la vulnerabilidad de las especies en México, se calculó el área de distribución actual 
por especie que se encuentra dentro de las áreas protegidas (AP) de cualquier categoría (IUCN y UNEP 2014) y dentro de los dos ecosistemas más amenazados de México: el bosque mesófilo de montaña y bosque tropical perennifolio (Challenger y Soberón 2008; selvas altas y medianas perennifolias y subperennifolias según INEGI 2014). Se incluyeron las categorías de riesgo de extinción para cada especie de acuerdo a los estándares del gobierno mexicano (SEMARNART 2010) e internacionales (IUCN 2013). Las distribuciones por especie se sumaron para presentar la riqueza taxonómica en México; debido al número de especies, esto solo se hizo para la familia Soricidae (Figura 1). Con la finalidad de mostrar el control de calidad sobre los datos de presencia con los que se construyeron los modelos, se muestra el intervalo altitudinal de los registros (Tabla 3 ) y sus valores de temperatura promedio anual y la precipitación anual (bio01, bio12; http://www. worldclim.org) representados en una gráfica de dispersión, junto con los valores climáticos para México y para el área en la que se calibró el modelo (Apéndice 1).

\section{Resultados}

Taxonomía y nomenclatura. Se presenta una lista con tres especies de topos y 36 de musarañas registradas en México, lo que representa el $8 \%$ y $9 \%$ de la diversidad mundial para cada grupo, respectivamente. Nuestra propuesta de las 39 especies alistadas es similar a las listas recientes (Ceballos y Arroyo-Cabrales 2013; Ramírez-Pulido et al. 2014), más la inclusión de una especie nueva de musaraña descrita recientemente (Guevara et al. 2014b). En contraste con Ceballos y Arroyo-Cabrales (2013) y de acuerdo con Ramírez-Pulido et al. (2014), consideramos a Cryptotis griseoventris y a Sorex saussurei como endémicas de México y a C. tropicalis con distribución hasta Guatemala (Woodman et al. 2012). El 67 \% de las especies son endémicas de México (Tabla 3). Para la familia Talpidae se sigue la propuesta de Yates y Salazar-Bravo (2005) que reconocen como especie válida a Scapanus anthonyi, anteriormente considerada como una subespecie de S. latimanus. Con esto, S. latimanus está representado en México por la subespecie S. I. ocultus. Por su parte, Scalopus aquaticus posee a las subespecies S. a. inflatus y S. a. montanus. En cuanto a la familia Soricidae, se trata al género Cryptotis como masculino (ICZN 2006), por lo que los nombres válidos para $C$. magna, C. mexicana, C. obscura y C. parva son: C. magnus, C. mexicanus, C. obscurus y $C$. parvus, respectivamente. Se integra al listado a Cryptotis lacandonensis, una especie nueva descrita de la Selva Lacandona en el sureste tropical mexicano (Guevara et al. 2014b). Mantenemos a C. parvus pueblensis como una subespecie (Choate 1970; Carraway 2007; Ceballos y Arroyo-Cabrales 2013), aunque existen menciones para ser reconocida a nivel específico (Woodman y Croft 2005; Guevara y Cervantes 2014). Con base en el artículo 31.2.1 del ICZN (1999), la terminación cola como parte de un sustantivo es retenida sin importar que el género con el que combina sea femenino o masculino; por lo anterior, los nombres válidos son C. alticola y S. monticola (ver Woodman 2012). Para el género Sorex se consideran las propuestas de Matson y McCarthy (2005), Carraway (2007) y Woodman et al. (2012).

Información geográfica y ejemplares de museo. El análisis del sesgo en el muestreo para las musarañas indicó que existe una tendencia significativa a colectarlas cerca de los caminos más cercanos $(X=3.78 \mathrm{~km}, Z=4.38, P<0.001)$. En este sentido, en las regiones en donde disminuye la red de carreteras como en el Istmo de Tehuantepec 0 en la Península de Baja California se observa una menor densidad de puntos de colecta. En cuanto al número de ejemplares, algunas especies del orden se conocen por tres o 
menos ejemplares colectados en México, como es el caso del topo Scalopus aquaticus y de las musarañas C. lacandonensis, C. merriami, Notiosorex cockrumi, N. villai, Sorex arizonae y S. stizodon (Tabla 3). Cabe destacar que para Scalopus aquaticus solo se recopilaron dos registros en México, siendo el ejemplar más reciente uno obtenido en 1950. En el caso de las musarañas, los registros más recientes para C. merriami y Sorex sclateri en México son de 1965, para N. cockrumi y S. macrodon de 1975 y para S. stizodon el único registro fue obtenido en 1895. Las especies con el menor número de localidades ( $\leq 5$ localidades) son Scapanus anthonyi, C. lacandonensis, C. nelsoni, $N$. cockrumi, N. villai, Sorex emarginatus, S. macrodon, S. orizabae, S. sclateri y S. stizodon. En el otro extremo están los taxones que han sido ampliamente colectados en el país, encabezando la lista las musarañas C. mexicanus, C. parvus, S. salvini, C. obscurus, S. saussurei, C. goldmani, S. veraepacis y C. magnus.

Tabla 1. Prueba de Jackknife para los modelos de nicho de 17 especies de musarañas de México de los géneros Cryptotis, Megasorex Notiosorex y Sorex con y sin información del sesgo en el muestreo.

\begin{tabular}{|c|c|c|c|c|c|c|}
\hline & \multirow{2}{*}{$n$} & \multicolumn{2}{|c|}{ Con sesgo } & \multicolumn{2}{|c|}{ Sin sesgo } & \multirow[b]{2}{*}{ Variables ambientales } \\
\hline & & éxito & P-valor & éxito & P-valor & \\
\hline C. alticola & 19 & 91.7 & $0.000^{*}$ & 94.7 & $0.000^{*}$ & bio01, bio02, bio03, bio04, bio07, bio12, bio15, bio 18 , pen \\
\hline C. goodwini & 16 & 84.6 & $0.000^{*}$ & 93.3 & $0.000^{*}$ & $\begin{array}{l}\text { bio01, bio03, bio } 04 \text {, bio } 05 \text {, bio12, bio } 15 \text {, bio } 17 \text {, bio } 18 \text {, } \\
\text { bio19, pen }\end{array}$ \\
\hline C. griseoventris & 5 & 80.0 & $0.000^{*}$ & 80.0 & $0.000^{*}$ & $\begin{array}{l}\text { bio01, bio03, bio } 04 \text {, bio05, bio07, bio12, bio15, bio } 18 \text {, } \\
\text { bio19, pen }\end{array}$ \\
\hline C. magnus & 25 & 94.1 & $0.000^{*}$ & 96.0 & $0.000^{*}$ & bio01, bio04, bio05, bio07, bio12, bio15, pen \\
\hline C. mayensis & 25 & 82.6 & $0.000^{*}$ & 83.3 & $0.000^{*}$ & bio01, bio03, bio04, bio05, bio12, bio15, bio18, pen \\
\hline C. merriami & 16 & 85.7 & $0.000^{*}$ & 85.7 & $0.000^{*}$ & $\begin{array}{l}\text { bio01, bio02, bio03, bio04, bio06, bio12, bio15, bio } 18 \text {, } \\
\text { bio19, pen }\end{array}$ \\
\hline C. peregrina & 5 & 75.0 & $0.000^{*}$ & 80.0 & $0.000^{*}$ & $\begin{array}{l}\text { bio } 01 \text {, bio } 02 \text {, bio } 03 \text {, bio } 04 \text {, bio } 05 \text {, bio } 12 \text {, bio } 14 \text {, bio } 15 \text {, } \\
\text { bio } 18 \text {, bio19, pen }\end{array}$ \\
\hline C.phillipsii & 21 & 81.3 & $0.000^{*}$ & 88.9 & $0.000^{*}$ & $\begin{array}{l}\text { bio01, bio } 02 \text {, bio } 03 \text {, bio } 04 \text {, bio } 05 \text {, bio12, bio14, bio } 15 \text {, } \\
\text { bio18, bio19, pen }\end{array}$ \\
\hline C. tropicalis & 8 & 87.5 & 0.225 & 87.5 & 0.396 & $\begin{array}{l}\text { bio01, bio02, bio03, bio05, bio07, bio12, bio14, bio15, } \\
\text { bio18, bio19, pen } \\
\text { bio01, bio02, bio03, bio04, bio05, bio12, bio14, bio15, }\end{array}$ \\
\hline M. gigas & 23 & 76.2 & $0.000^{*}$ & 87.0 & $0.000^{*}$ & $\begin{array}{l}\text { bio18, bio19, pen } \\
\text { bio01, bio02, bio03, bio05, bio07, bio12, bio15, bio17, }\end{array}$ \\
\hline N. evotis & 19 & 66.7 & $0.015^{*}$ & 63.1 & $0.000^{*}$ & $\begin{array}{l}\text { bio18, bio19, pen } \\
\text { bio01, bio02, bio03, bio07, bio10, bio12, bio14, bio15, }\end{array}$ \\
\hline S. arizonae & 18 & 94.1 & $0.000^{*}$ & 94.1 & $0.000^{*}$ & bio19, pen \\
\hline S. ixtlanensis & 10 & 90.0 & $0.000^{*}$ & 60.0 & $0.002^{*}$ & bio01, bio02, bio03, bio05, bio12, bio14, bio15, bio18, pen \\
\hline S. mediopua & 6 & 75.0 & 0.114 & 83.3 & 0.155 & $\begin{array}{l}\text { bio01, bio } 02 \text {, bio } 03 \text {, bio04, bio } 06 \text {, bio12, bio14, bio } 15 \text {, } \\
\text { bio18, pen }\end{array}$ \\
\hline S. milleri & 6 & 50.0 & $0.000^{*}$ & 50.0 & $0.000^{*}$ & $\begin{array}{l}\text { bio } 01 \text {, bio } 02 \text {, bio } 03 \text {, bio10, bio11, bio12, bio14, bio } 15 \text {, } \\
\text { bio16, pen }\end{array}$ \\
\hline Soreopolus & 11 & 88.9 & $0.000^{*}$ & 90.0 & $0.000^{*}$ & $\begin{array}{l}\text { bio } 01 \text {, bio02, bio03, bio04, bio05, bio12, bio14, bio } 15 \text {, } \\
\text { bio18, pen }\end{array}$ \\
\hline S. ventralis & 22 & 91.0 & $0.000^{*}$ & 93.8 & $0.000^{*}$ & $\begin{array}{l}\text { bio01, bio02, bio03, bio04, bio06, bio12, bio14, bio15, } \\
\text { bio18, pen }\end{array}$ \\
\hline
\end{tabular}


Modelos de nicho ecológico. En 15 de las 17 especies analizadas bajo el método de Jackknife por su pequeño número de muestra (Pearson et al. 2007), los resultados predictivos fueron significativos (Tabla 1). En todos los casos el porcentaje de registros correctamente predichos fue mayor al 75\%, excepto para Sorex milleri y Notiosorex evotis (Tabla 1). Las especies con predicciones no diferentes a lo esperado al azar fueron C. tropicalis y S. mediopua, por lo que no se propone una estimación de su distribución actual. Una posible explicación a este resultado, además del tamaño de muestra, es que en ambas especies los datos de colecta muestran una notable variación en el espacio ambiental, dificultando la estimación del nicho ecológico (Figura 2). Para el caso de las especies con $\geq 26$ registros (Tabla 2), todos los modelos mostraron valores relativamente altos de $\mathrm{AUC}_{\mathrm{ev}}(>0.87)$, excepto para el caso del topo Scalopus aquaticus $\left(A \cup C_{e v}=0.821\right)$ y valores de $A U C_{d i}$ fueron bajos $(0.014-0.041)$, lo cual sugiere que los modelos no están sobre ajustados a los datos de calibración (Warren y Seifter 2011). Cuando comparamos los modelos realizados con y sin el archivo del sesgo en el muestreo, no encontramos tendencia hacia la mejora en la evaluación de los modelos para alguno de ellos (Tablas 1 y 2). Lo mismo ocurrió al inspeccionar los MNE proyectados en el espacio geográfico.

Tabla 2. Evaluación de modelos de nicho de dos especies de topos de los géneros Scalopus y Scapanus y 10 especies de musarañas de los géneros Cryptotis, Notiosorex y Sorex de México con y sin información del sesgo en el muestreo.

\begin{tabular}{|c|c|c|c|c|c|c|}
\hline & \multirow{2}{*}{$n$} & \multicolumn{2}{|c|}{ Con sesgo } & \multicolumn{2}{|c|}{ Sin sesgo } & \multirow[b]{2}{*}{ Variables ambientales } \\
\hline & & $\mathrm{AUC}_{\mathrm{ev}}$ & $A \cup C_{d i}$ & $A \cup C_{e v}$ & $A \cup C_{d i}$ & \\
\hline \multicolumn{7}{|l|}{ Talpidae } \\
\hline S. aquaticus & 804 & & & $\begin{array}{c}0.821 \pm \\
0.043\end{array}$ & 0.034 & $\begin{array}{l}\text { bio } 01 \text {, bio } 02 \text {, bio } 05 \text {, bio11, bio12, bio14, bio } 15 \text {, } \\
\text { bio } 19 \text {, pen }\end{array}$ \\
\hline S. latimanus & 595 & & & $\begin{array}{c}0.904 \pm \\
0.039\end{array}$ & 0.018 & $\begin{array}{l}\text { bio01, bio05, bio07, bio11, bio12, bio14, bio17, } \\
\text { bio19, pen }\end{array}$ \\
\hline \multicolumn{7}{|l|}{ Soricidae } \\
\hline C. goldmani & 30 & $\begin{array}{c}0.978 \pm \\
0.052\end{array}$ & 0.006 & $\begin{array}{c}0.961 \pm \\
0.059\end{array}$ & 0.012 & bio01, bio05, bio07, bio12, bio15, bio18, pen \\
\hline C. mexicanus & 93 & $\begin{array}{c}0.889 \pm \\
0.107\end{array}$ & 0.028 & $\begin{array}{c}0.908 \pm \\
0.101\end{array}$ & 0.031 & $\begin{array}{l}\text { bio01, bio02, bio05, bio07, bio12, bio14, bio15, } \\
\text { bio18, pen }\end{array}$ \\
\hline C. obscurus & 42 & $\begin{array}{c}0.948 \pm \\
0.118\end{array}$ & 0.021 & $\begin{array}{c}0.953 \pm \\
0.091\end{array}$ & 0.015 & $\begin{array}{l}\text { bio } 01 \text {, bio } 02 \text {, bio } 03 \text {, bio } 05 \text {, bio } 06 \text {, bio } 07 \text {, bio } 12 \text {, } \\
\text { bio } 15 \text {, pen }\end{array}$ \\
\hline C.parvus & 660 & $\begin{array}{c}0.875 \pm \\
0.057\end{array}$ & 0.021 & $\begin{array}{l}0.87 \pm \\
0.066\end{array}$ & 0.021 & $\begin{array}{l}\text { bio } 01 \text {, bio } 02 \text {, bio } 03 \text {, bio } 05 \text {, bio } 08 \text {, bio } 12 \text {, bio } 15 \text {, } \\
\text { bio } 17 \text {, bio } 18 \text {, pen }\end{array}$ \\
\hline N. crawfordi & 260 & $\begin{array}{c}0.871 \pm \\
0.126\end{array}$ & 0.041 & $\begin{array}{c}0.874 \pm \\
0.123\end{array}$ & 0.035 & $\begin{array}{l}\text { bio01, bio02, bio03, bio07, bio08, bio09, bio12, } \\
\text { bio13, bio17, bio19, pen }\end{array}$ \\
\hline S. monticola & 764 & $\begin{array}{l}0.906 \pm \\
0.036\end{array}$ & 0.014 & $\begin{array}{c}0.911 \pm \\
0.031\end{array}$ & 0.010 & $\begin{array}{l}\text { bio01, bio02, bio03, bio } 07 \text {, bio08, bio12, bio } 14 \text {, } \\
\text { bio15, bio18, pen }\end{array}$ \\
\hline S. ornatus & 212 & $\begin{array}{c}0.942 \pm \\
0.045\end{array}$ & 0.018 & $\begin{array}{c}0.944 \pm \\
0.042\end{array}$ & 0.017 & $\begin{array}{l}\text { bio01, bio02, bio03, bio07, bio08, bio09, bio10, } \\
\text { bio12, bio15, bio18, pen }\end{array}$ \\
\hline S. saussurei & 38 & $\begin{array}{l}0.917 \\
\pm 0.1\end{array}$ & 0.028 & $\begin{array}{c}0.931 \pm \\
0.096\end{array}$ & 0.021 & $\begin{array}{l}\text { bio01, bio02, bio03, bio } 04 \text {, bio } 07 \text {, bio } 12 \text {, bio } 15 \text {, } \\
\text { bio } 18 \text {, pen }\end{array}$ \\
\hline S. salvini & 64 & $\begin{array}{c}0.948 \pm \\
0.084\end{array}$ & 0.014 & $\begin{array}{c}0.946 \pm \\
0.094\end{array}$ & 0.015 & $\begin{array}{l}\text { bio } 01 \text {, bio } 02 \text {, bio03, bio } 05 \text {, bio } 12 \text {, bio } 14 \text {, bio } 15 \text {, } \\
\text { bio18, bio19, pen }\end{array}$ \\
\hline S. veraepacis & 50 & $\begin{array}{c}0.935 \\
\pm 0.076\end{array}$ & 0.014 & $\begin{array}{c}0.945 \pm \\
0.065\end{array}$ & 0.017 & $\begin{array}{l}\text { bio01, bio02, bio03, bio } 05 \text {, bio12, bio14, bio } 18 \text {, } \\
\text { bio19, pen }\end{array}$ \\
\hline
\end{tabular}

$n=$ número de localidades; $A \cup C_{\mathrm{ev}}$ : $\mathrm{AUC}$ de datos de evaluación; $\mathrm{AUC}_{\mathrm{di}}$ : diferencia de $\mathrm{AUC}$ entre datos de calibración y de evaluación. Se muestran las variables ambientales utilizadas por especie (ver Materiales y Métodos) 
La suma de los modelos de distribución encontró 11,305 km² predichos como habitables para la familia Talpidae y $1,012,197 \mathrm{~km}^{2}$ para la familia Soricidae dentro de México (Tabla 3, Apéndice 1). Las especies con la mayor superficie de distribución actual son las musarañas Notiosorex crawfordi, Cryptotis parvus, N. evotis, Sorex ventralis y C. mayensis (> 125,000 km²), mientras que las especies endémicas de México que poseen distribución más restringida son S. milleri, C peregrina, C. griseoventris y $C$. magnus ( $<5,000 \mathrm{~km}^{2}$, Tabla 3 ). El $75.6 \%$ de la superficie estimada para la familia Soricidae es adecuada para al menos una especie, localizándose principalmente en las zonas desérticas del norte de México y en la Península de Yucatán, en donde se extienden las distribuciones de N. crawfordi, C. parvus y C. mayensis, principalmente. Mientras tanto, la región que podría albergar a la mayor riqueza taxonómica se encuentra en las zonas altas del centro y sur del país al estimar que el $5.89 \%$ de la superficie es habitable incluso para cinco a nueve especies de los géneros Cryptotis y Sorex (Figura 1), como las especies endémicas de México C. alticola, C. goldmani, C. griseoventris, C. magnus, C. mexicanus, C. peregrina, C. phillipsii, Sorex ixtlanensis, S. oreopolus, S. saussureri y S. ventralis; además de S. macrodon, S. mediopua, S. orizabae, S. sclateri y $S$. ventralis que a pesar de no haber estimado su distribución, también habitan esas zonas.

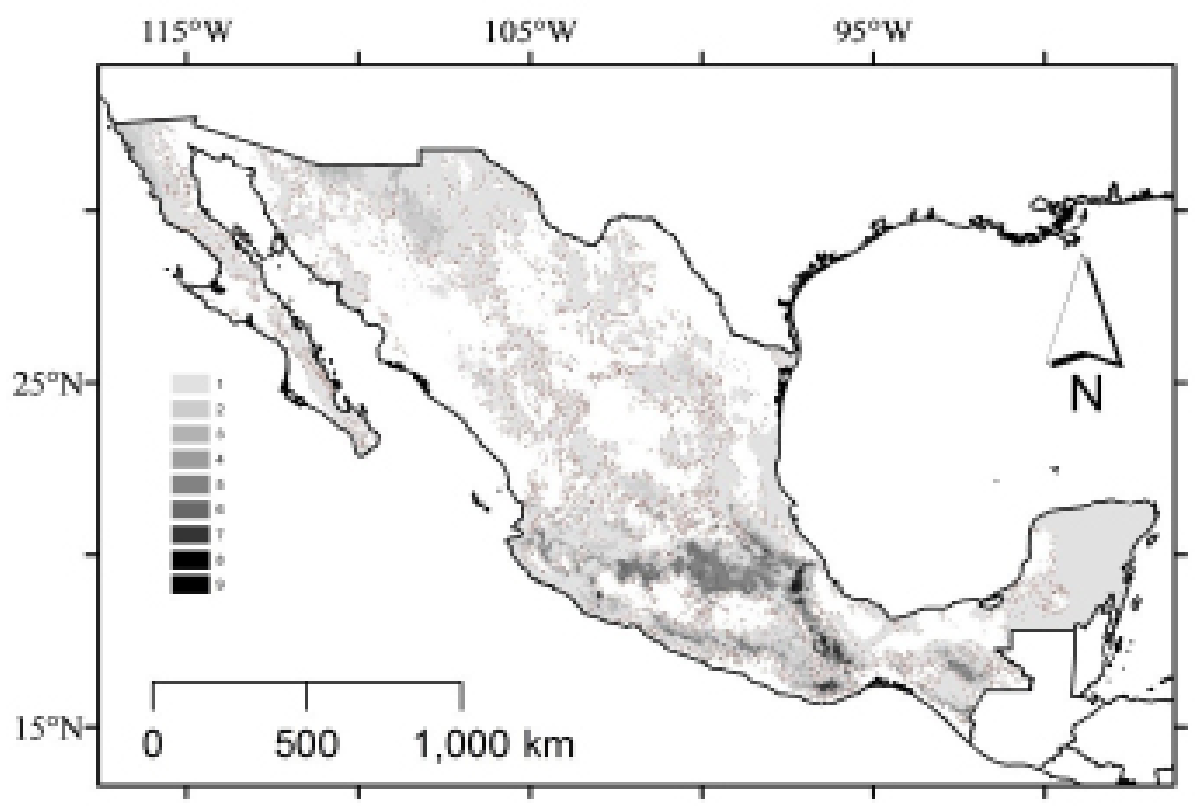

Figura 1. Riqueza de especies de musarañas (orden Eulipotyphla) en México. Las zonas más oscuras muestran la distribución actual estimada para un mayor número de especies ( 1 a 9 especies).

Al contabilizar el hábitat transformado por el hombre dentro de la distribución predicha como habitable, las especies con el mayor porcentaje de afectación son el topo Scalopus latimanus y las musarañas Cryptotis merriami, C. mexicanus, Sorex ornatus y C. obscurus (Tabla 3). Por otro lado, las especies con menor proporción de suelo transformado son las musarañas S. milleri, C. peregrina, S. arizonae, S. montícola, C. magnus y C. goldmani. Cabe mencionar que el cálculo del hábitat transformado fue conservador, ya que no se consideró el rubro de áreas de uso en agricultura, debido a que algunas especies pueden tolerar esta transformación (Cervantes et al. 2008; Guevara et al. 2011). De ser incluidas, el área de distribución actual de las especies podría ser menor. 
Tabla 3. Número de ejemplares de topos y musarañas colectados en México ( $n$ ), superficie de la distribución actual y porcentaje dentro de hábitat transformado, en áreas protegidas (AP) y en ecosistemas terrestres más amenazados de México (BMM, Bosque Mesófilo de Montaña; BTP, Bosque Tropical Perennifolio). Se presenta la categoría de riesgo de extinción (SEMARNAT 2010 y IUCN 2013) y el intervalo altitudinal de los registros (msnm). El fondo gris señala a las especies endémicas del país

\begin{tabular}{|c|c|c|c|c|c|c|c|c|}
\hline & $\varepsilon$ & 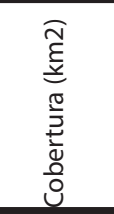 & 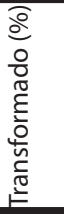 & 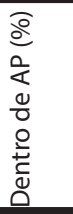 & $\begin{array}{l}\stackrel{\jmath}{0} \\
\sum_{\infty}^{\infty} \\
\sum_{\infty}^{\lambda}\end{array}$ & $\underset{\succeq}{\beth}$ & $\begin{array}{l}\text { 这 } \\
\sum \\
\sum_{\text {它 }}\end{array}$ & 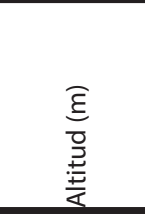 \\
\hline \multicolumn{9}{|l|}{ FAMILIA TALPIDAE (topos) } \\
\hline Scalopus aquaticus (Linnaeus, 1758) & 2 & 4,761 & 3.9 & 14.2 & 0.0 & LC & $\mathrm{P}$ & $0-1333$ \\
\hline Scapanus anthonyi J. A. Allen, 1893 & 12 & - & - & - & - & - & A & $1712-2389$ \\
\hline Scapanus latimanus (Bachman, 1842) & 7 & 6,544 & 8.4 & 0.4 & 0.0 & LC & $P$ & $0-2801$ \\
\hline \multicolumn{9}{|l|}{ FAMILIA SORICIDAE (musarañas) } \\
\hline Cryptotis alticola (Merriam, 1895) & 60 & 63,170 & 3.6 & 19.3 & 1 & - & PR & $2242-4134$ \\
\hline Cryptotis goldmani (Merriam, 1895) & 116 & 41,824 & 0.9 & 1.6 & 6.3 & LC & PR & $961-3433$ \\
\hline Cryptotis goodwini Jackson, 1933 & 4 & 1,546 & 5.0 & 52.1 & 32.5 & LC & - & $1464-3085$ \\
\hline Cryptotis griseoventris Jackson, 1933 & 21 & 3,067 & 1.5 & 1.6 & 0.2 & V & - & $1912-2775$ \\
\hline Cryptotis lacandonensis Guevara et al., 2014 & 2 & - & - & - & - & - & - & 90 \\
\hline Cryptotis magnus (Merriam, 1895) & 105 & 3,610 & 0.6 & 8.0 & 20.6 & V & PR & $1300-3109$ \\
\hline Cryptotis mayensis (Merriam, 1901) & 82 & 125,079 & 2.3 & 19.9 & 11.2 & LC & PR & $0-600$ \\
\hline Cryptotis merriami Choate, 1970 & 1 & 16,431 & 7.9 & 15.9 & 15.6 & $\mathrm{LC}$ & - & $438-1659$ \\
\hline Cryptotis mexicanus (Coues, 1877) & 461 & 41,509 & 6.9 & 7.2 & 10.3 & $\mathrm{LC}$ & - & $435-3150$ \\
\hline Cryptotis nelsoni (Merriam, 1895) & 56 & - & - & - & - & CE & PR & $932-1426$ \\
\hline Cryptotis obscurus (Merriam, 1895) & 160 & 6,326 & 6.3 & 19.2 & 10.2 & V & PR & $790-2615$ \\
\hline Cryptotis parvus (Say, 1823) & 396 & 332,387 & 4.6 & 10.4 & 3.3 & LC & PR & $0-2532$ \\
\hline Cryptotis peregrina (Merriam, 1895) & 44 & 2,205 & 0.2 & 4.1 & 4.3 & - & PR & $2559-3056$ \\
\hline Cryptotis phillipsii (Schaldach, 1966) & 53 & 5,780 & 2.0 & 0.8 & 4.7 & V & - & $885-2417$ \\
\hline Cryptotis tropicalis (Merriam, 1895) & 14 & - & - & - & - & - & - & $444-1594$ \\
\hline Megasorex gigas (Merriam, 1897) & 59 & 61,335 & 3.1 & 8.3 & 0.9 & $\mathrm{LC}$ & A & $706-1412$ \\
\hline Notiosorex cockrumi Baker et al., 2003 & 2 & - & - & - & - & LC & A & $888-1475$ \\
\hline Notiosorex crawfordi (Coues, 1877) & 86 & 388,464 & 1.3 & 13.4 & 0.02 & LC & A & $15-2323$ \\
\hline Notiosorex evotis (Coues, 1877) & 38 & 143,899 & 2.9 & 8.8 & 0.3 & $\mathrm{LC}$ & A & $2-1856$ \\
\hline Notiosorex villai Carraway y Timm, 2000 & 3 & - & - & - & - & V & A & $750-1258$ \\
\hline Sorex arizonae Diersing y Hoffmeister, 1977 & 1 & 42,061 & 0.5 & 14.7 & 0.0 & LC & $P$ & $1470-2435$ \\
\hline Sorex emarginatus Jackson, 1925 & 8 & - & & - & - & LC & - & $1757-2496$ \\
\hline Sorex ixtlanensis Carraway, 2007 & 38 & 16,905 & 4.4 & 3.4 & 23.3 & - & & $1185-2886$ \\
\hline Sorex macrodon Merriam, 1895 & 11 & - & - & - & - & $\mathrm{V}$ & $A$ & $752-1697$ \\
\hline Sorex mediopua Carraway, 2007 & 39 & - & - & - & - & $\mathrm{LC}$ & - & $1480-2973$ \\
\hline Sorex milleri Jackson, 1947 & 51 & 611 & 0.0 & 22.6 & 0.0 & V & PR & $2521-3573$ \\
\hline Sorex monticola Merriam, 1890 & 15 & 22,794 & 0.5 & 9.3 & 0.0 & LC & A & $495-4027$ \\
\hline Sorex oreopolus Merriam, 1892 & 17 & 52,222 & 5.6 & 19.3 & 0.9 & LC & - & $2243-3823$ \\
\hline Sorex orizabae Merriam 1895 & 30 & - & - & - & - & LC & - & $2103-3557$ \\
\hline Sorex ornatus Merriam, 1895 & 50 & 14,259 & 6.7 & 9.9 & 0.0 & LC & A & $0-2659$ \\
\hline Sorex saussurei Merriam, 1892 & 136 & 53,042 & 4.3 & 21.4 & 1.4 & LC & - & $1733-3941$ \\
\hline Sorex sclateri Merriam, 1897 & 5 & - & - & - & - & CE & A & $1341-1483$ \\
\hline Sorex stizodon Merriam, 1895 & 1 & - & - & - & - & CE & A & 2130 \\
\hline Sorex ventralis Merriam, 1895 & 86 & 131,738 & 3.6 & 12.4 & 1.7 & LC & - & $1571-4129$ \\
\hline Sorex salvini Merriam, 1897 & 234 & 95,791 & 1.5 & 13.2 & 4.1 & LC & $A$ & $1403-3941$ \\
\hline Sorex veraepacis Alston, 1877 & 109 & 29,980 & 4.5 & 8.9 & 16.4 & $\mathrm{LC}$ & $A$ & $907-3162$ \\
\hline
\end{tabular}


El $63 \%$ de las especies del orden poseen registros confirmados dentro de las Áreas Protegidas (AP), mientras que los modelos sugieren que todas las especies en las que se estimó su distribución actual habitan dentro de las AP. Del total de la superficie estimada, el topo Scapanus latimanus y las musarañas C. goldmani, C. griseoventris y C. phillipsii tienen menos del $2 \%$ de sus distribuciones dentro de las AP; por el otro lado, la especie con la mayor proporción en AP es C. goodwini (52.1\%), aunque su distribución en México es relativamente pequeña $\left(<1,546 \mathrm{~km}^{2}\right)$. En referencia a los dos ecosistemas terrestres más amenazados del país (Challenger y Soberón 2008), $C$. goodwini (32.5\%), Sorex ixtlanensis (23.3\%) y C. magnus (20.6\%), son las especies que poseen la mayor distribución actual dentro de los bosques mesófilos de montaña y los bosques tropicales perennifolios. A estas especies habrá que sumar otras más para las que no se estimó su distribución, pero sus registros se ubican en estos ecosistemas, como las musarañas C. lacandonensis, C. nelsoni, S. sclateri y S. stizodon.

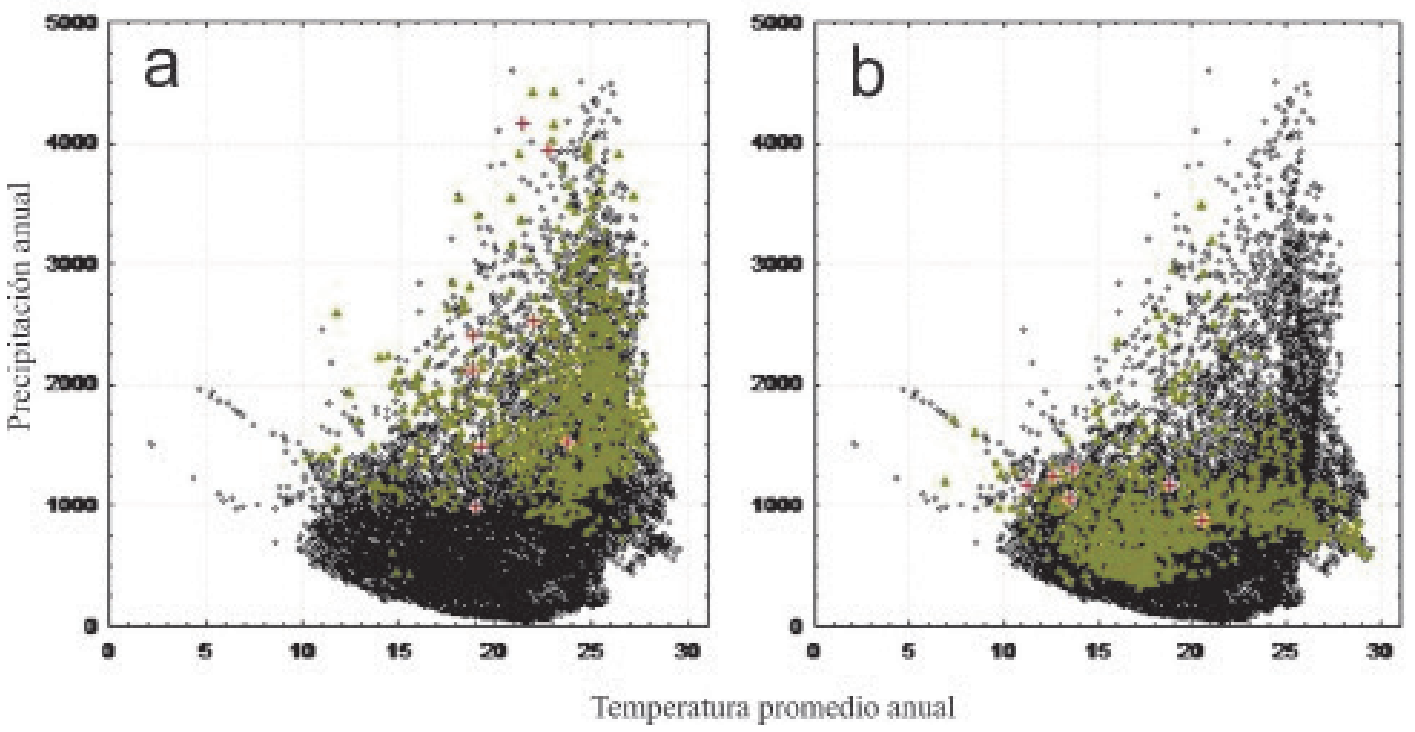

Figura 2. Gráfica de dispersión de las localidades de colecta (cruces rojas) de Cryptotis tropicalis (a) y Sorex mediopua (b) en el espacio ambiental. Los puntos negros son los valores para México y los puntos verdes los valores para el área de calibración del modelo.

\section{Discusion}

Sumado a las revisiones de Maldonado (1999) y Carraway (2007), los resultados de este estudio representan el mayor esfuerzo integral por dar a conocer el estado del conocimiento sobre el orden Eulipotyphla en México. Actualmente, 11 de las 39 especies del orden poseen menos de 10 ejemplares de museo colectados dentro del país. Si bien es cierto que esto puede deberse a la dificultad que implica la captura de topos y musarañas, la cantidad de registros también podría indicar el estado de riesgo de las especies (Escalante-Espinosa 2003). Cualquiera que fuese el caso, la colecta científica sigue siendo un aspecto esencial para la adquisición y validación de datos que van desde la taxonomía alfa hasta el manejo y la toma de decisiones en conservación (Sánchez-Cordero et al. 2001; Patterson 2002; Rocha et al. 2014). Al menos antes del inicio del presente siglo, la colecta de topos y musarañas en México podría definirse como contingente, ya que era un resultado circunstancial al tratar de colectar diversas especies de roedores (Ramírez-Pulido et al. 2004; Carraway 2007). 
En el caso de las musarañas, sólo los esfuerzos más recientes han explotado el uso de técnicas de bajo precio para colectarlas, como las trampas de caída o tipo "pitfall", aunque sólo se ha realizado en contadas regiones del país (Cervantes et al. 2008; Guevara et al. 2011).

Es posible que algunas especies de eulipotiflos que no han sido colectadas durante las últimas décadas en México, ya se encuentren extirpadas o extintas (Ceballos y Navarro 1991). El caso más alarmante es la musaraña de San Cristóbal de Las Casas, Sorex stizodon que sólo se conoce del holotipo colectado en 1895 (Goldman 1951; Naranjo y Espinoza 2001). Otro caso ejemplar es el del topo Scalopus aquaticus, ya que el último registro confirmado dentro del país tiene más de medio siglo y el estado de conservación de sus poblaciones es desconocido (Ceballos y Navarro 1991). Un caso diferente, donde se ha redescubierto a una especie, lo representa la musaraña Cryptotis nelsoni, ya que sólo se conocía de la serie tipo colectada en 1894, pero más de un siglo después se obtuvieron nuevos ejemplares y más localidades de colecta en la Sierra de Los Tuxtlas, en la costa este de México (Cervantes y Guevara 2010).

Con base en la distribución actual, el número de registros, la protección actual dentro de AP y/o las amenazas potenciales, consideramos que las musarañas Cryptotis griseoventris y la recientemente descrita $C$. lacandonensis deberían estar protegidas por el gobierno mexicano. Actualmente, C. griseoventris se encuentra catalogada como vulnerable por las normas internacionales, debido a su distribución restringida y a que su área de distribución está severamente fragmentada debido a la extensa deforestación (IUCN 2013; Guevara et al. 2014a). Otras especies también enfrentan elevadas tasas de deforestación dentro de sus áreas históricas como S. macrodon, en la que se ha estimado que solo permanece alrededor del $15 \%$ de la cobertura forestal original en su distribución (Sánchez-Cordero et al. 2005). Las zonas con mayor riqueza taxonómica y mayor número de endemismos del orden coincide con regiones de particular importancia en la conservación de los mamíferos en México como la Faja Volcánica Transmexicana, la Sierra Madre del Sur y Los Altos de Chiapas (Escalante-Espinosa 2003; Vázquez et al. 2009). Estas regiones poseen actualmente extensas áreas que han sido transformadas para su uso en la agricultura y el urbanismo (Sánchez-Cordero et al. 2005, 2009; PACC 2011). Las especies endémicas que se distribuyen en estas regiones serían las más vulnerables, como las musarañas Cryptotis alticola, C. goldmani, C. griseoventris, C. phillipsii, Sorex oreopolus, S. saussurei, S. sclateri, S. stizodon y S. ventralis. Todas las especies del género Cryptotis de estas zonas se encuentran en alguna categoría de riesgo federal o internacional (Tabla 3), mientras que para el género Sorex, solo S. sclateri, y S. stizodon están bajo alguna categoría de riesgo (Semarnat 2010; IUCN 2013).

El cambio climático también podría ser una amenaza para los topos y las musarañas en México, sin olvidar que cada especie reacciona de manera particular (Peterson et al. 2001; Trejo et al. 2011; Schloss et al. 2012). Estimaciones recientes sugieren que cerca del $68 \%$ de la extensión de los bosques mesófilos en México se perderán durante el presente siglo como una consecuencia del cambio climático (Ponce-Reyes et al. 2012); de cumplirse este escenario, al menos 24 de las 36 especies de musarañas perderán el hábitat adecuado para su sobrevivencia. Para el caso de los boques tropicales perennifolios, el cambio climático será responsable de una pérdida cercana al $80 \%$ en la distribución para la musaraña Megasorex gigas (Trejo et al. 2011), representante de un género monotípico y uno de los 12 géneros de mamíferos endémicos de México. 
Es importante mencionar que, los estudios recientes en torno a la familia Soricidae en México han contribuido a actualizar el conocimiento sobre la riqueza y distribución actual de las especies (Carraway 2007; Cervantes et al. 2008; Cervantes y Guevara 2010; Guevara et al. 2014b) y a incrementar el conocimiento sobre su variación morfológica intra e interespecífica (Ramírez-Pulido et al. 2004, 2005; Carraway 2007). Como lo indican estudios previos y como lo sugiere la distribución fragmentada en diversas especies de los géneros Cryptotis y Sorex (Apéndice 1), aún se requieren evaluaciones taxonómicas para esclarecer el límite taxonómico entre algunas especies (Esteva et al. 2010; Guevara y Cervantes 2014). Para el caso de la familia Talpidae ha habido un escaso conocimiento generado durante las últimas décadas y se conoce poco sobre el estado de las poblaciones (Ceballos y Navarro 1991), por lo que el paso inmediato a seguir es poner en claro la situación actual de las poblaciones de las tres especies de topo.

A pesar del sesgo significativo de colecta a lo largo de los caminos como en el caso de las musarañas, la comparación entre los MNE que consideran el sesgo contra los que no lo consideran, muestran que los resultados son similares. Esto podría indicar que el sesgo geográfico en el muestreo de este grupo de mamíferos no ha arrastrado un sesgo ambiental. No obstante, otras aproximaciones que evalúan el sesgo geográfico para ser incorporado en el MNE podrían ser exploradas en el futuro (Phillips et al. 2009; Anderson y González Jr. 2011). En cuanto al número de muestra, infortunadamente la mayoría de la especies de eulipotiflos en México poseen un número reducido de localidades, por lo que en esos casos las estimaciones deberían tomarse con mayor cautela, a pesar de haber elegido el método más apropiado (Pearson et al. 2007). Conforme se incremente el número de localidades, será posible validar y robustecer los resultados para especies con tamaños de muestra reducidos (Jackson y Robertson 2011). Consideramos que los esfuerzos de colecta deberían enfocarse a los taxones menos representados en las colecciones biológicas y en aquellos que no han sido registrados en décadas. Un paso inicial podría ser el impulso de inventarios dirigido a estas especies, particularmente dentro de las AP (Sánchez-Cordero et al. 2005, 2009; Ceballos 2007; Rocha et al. 2014). Además, es importante utilizar métodos adecuados de colecta y dar prioridad a las regiones actualmente adecuadas para las especies (Apéndice 1; Raxworthy et al. 2003; Woodman et al. 2012). A medida que se obtengan más datos y se actualicen sobre los topos y las musarañas en México, las evaluaciones y los análisis subsecuentes podrán redefinir las prioridades de conservación dentro de uno de los grupos de mamíferos más diversos del país.

\section{Agradecimientos}

Los siguientes curadores y asistentes ofrecieron, en todo momento, las facilidades para examinar ejemplares en las colecciones bajo su cargo: F. X. González Cózatl y E. Arellano (CMC-CEAMISCH); A. Riechers (CZRMA); C. Lorenzo (ECO-SC-M); J. Arroyo Cabrales (INAH); R. M. Timm (KU); L. León Paniagua (MZFC); M. Briones Salas (OAXMA); S. Hernández Betancourt (UADY); J. Ramírez Pulido y N. González Ruíz (UAMI); N. Woodman y S. Peurach (USNM). S. Ortega García contribuyó invaluablemente en la depuración de la base de datos de la familia Soricidae. LG agradece al Posgrado en Ciencias Biológicas (UNAM) y al Consejo Nacional de Ciencias y Tecnología (CONACyT) por las becas y apoyos económicos para realizar los estudios de posgrado. 
La Comisión Nacional para el Conocimiento y Uso de la Biodiversidad aportó apoyo económico para la depuración de bases de datos y consulta de museos (CONABIO, FB1587/JM044/12).

\section{Resumen}

Introducción. Los topos (Talpidae) y las musarañas (Soricidae) son los únicos representantes del orden Eulipotyphla en México, conformando cerca del $7 \%$ de la mastofauna del país. A pesar de su riqueza, algunos aspectos básicos como la taxonomía alfa y el conocimiento de su distribución geográfica permanecen incompletos. La falta de esta información implica que el estado de conservación de los eulipotiflos también permanezca sin ser evaluado, lo que implica un serio impedimento para el diseño de estrategias de manejo para un grupo que tiende a ser especialmente susceptible al cambio climático y a los impactos en la transformación del hábitat. Por tal motivo, con base en la información de colecciones de historia natural y datos ambientales espaciales se realizó una evaluación del estado actual del conocimiento y las posibles amenazas a la supervivencia de los topos y musarañas mexicanos.

Metodología. Revisamos la información disponible en las colecciones biológicas, bases de datos y registros de la literatura. Evaluamos el sesgo geográfico de colecta y estimamos la distribución actual de casi todas las especies de topos y musarañas registrados en México mediante el modelado de sus nichos ecológicos, reteniendo áreas con hábitat natural remanente. Por último, calculamos la extensión de la distribución de cada especie dentro de las Áreas Protegidas y dentro de los ecosistemas más amenazados en México con la finalidad de identificar a los taxones más vulnerables.

Resultados. La diversidad mexicana de eulipotiflos está representada por tres especies de topos y 36 de musarañas. 26 de estas especies (67\%) son endémicas del país y 27 (69\%) se encuentran bajo alguna categoría de riesgo de acuerdo al gobierno mexicano o por instancias internacionales. Once taxones se conocen por no más de 10 ejemplares o por muy pocas localidades en el país. Una musaraña en particular (Sorex stizodon) no ha sido registrada desde hace más de un siglo. Debido al escaso número de localidades $(<5)$, no se estimó la distribución actual de 12 especies. La región que podría albergar a la mayor riqueza taxonómica se encuentra en las zonas altas del centro y sur del país. Las especies con el mayor porcentaje de hábitat transformado son el topo Scalopus latimanus y las musarañas Cryptotis merriami, C. mexicanus, C. obscurus y Sorex ornatus. Con base en la distribución actual, el número de registros, la protección actual dentro de AP y/o las amenazas potenciales, las musarañas Cryptotis griseoventris y la recientemente descrita C. lacandonensis deberían estar protegidas por el gobierno mexicano.

Discusión y conclusiones. Este trabajo proporciona la primera documentación detallada de la información disponible sobre la taxonomía, nomenclatura, distribución y amenazas de los topos y musarañas en México. La información obtenida en colecciones de historia natural corrobora el escaso y sesgado conocimiento sobre la distribución actual de los eulipotiflos (Ramírez-Pulido et al. 2005; Carraway 2007). Nuestro análisis espacial proporciona evidencia de que algunas especies podrían estar más amenazadas de lo que actualmente se reconoce por criterios globales (UICN) y por el gobierno mexicano. El bajo número de ejemplares $(<10)$ de algunos eulipotiflos podría ser consecuencia de la falta de muestreo. Conforme se incremente el número de localidades, será posible validar y robustecer los resultados de modelado de nicho ecológico para especies con tamaños de muestra reducidos. Nuestros modelos de nicho convertidos a distribución actual deberían ser utilizados para dirigir esfuerzos de estudios de campo y colecta científica, con el fin de incrementar la información sobre el estado actual de las poblaciones de topos y musarañas.

Palabras clave: colecciones biológicas, distribución actual, Insectivora, inventarios, mamíferos pequeños, sesgo a caminos.

\section{Literatura citada}

Anderson, R. P., E I. Gonzalez JR. 2011. Species-specific tuning increases robustness to sampling bias in models of species distributions: an implementation with Maxent. Ecological Modelling 222: 2796-2811.

Asher, R. J., y K. M. Helgen. 2010. Nomenclature and placental mammal phylogeny. BMC Evolutionary Biology 10:102. 
Carraway, L. N. 2007. Shrews (Eulypotyphla: Soricidae) of Mexico. Monographs of the Western North American Naturalist 3:1-91.

Ceballos, G. 2007. Conservation priorities for mammals in megadiverse Mexico: the efficiency of reserve networks. Ecological Applications 17:569-578.

Ceballos, G., y J. Arroyo-Cabrales. 2013. Lista actualizada de los mamíferos de México 2012. Revista Mexicana de Mastozoología, nueva época 2:27-80.

Ceballos, G., y D. Navarro. 1991. Diversity and conservation of Mexican mammals. Pp: 167198 en Latin American Mammalogy: history, diversity and conservation (Mares, M. A., y D. J. Schmidly, eds.). University of Oklahoma Press. Norman, EE.UU.

Cervantes, F. A., A. Montiel, y A. García. 2008. Shrews (Mammalia, Soricomorpha) from Colima, México. The Southwestern Naturalist 53:101-104.

Cervantes, F. A., y L. Guevara. 2010. Rediscovery of the critically endangered Nelson's small-eared shrew (Cryptotis nelsoni), endemic to Volcán San Martín, Eastern México. Mammalian Biology 75:451-454.

Challenger, A., y J. Soberón. 2008. Los ecosistemas terrestres. Pp. 87-108 en Capital natural de México, vol. I: Conocimiento actual de la biodiversidad. Comisión Nacional para el Conocimiento y Uso de la Biodiversidad. Ciudad de México, México.

ChoAte, J. 1970. Systematics and Zoogeographic of Middle American shrews of the genus Cryptotis. University of Kansas Publications. Museum of Natural History 19:195-317.

Churchfield, S. 2002. Why are shrews so small? The costs and benefits of small size in northern temperate Sorex species in the context of foraging habits and prey supply. Acta Theriologica 47:169-184.

Douady, C. J., P. I. Chatelier, O. Madsen, W. W. de Jong, F. Catzeflis, M. S. Springer, y M. J. Stanhope. 2002. Molecular phylogenetic evidence confirming the Eulipotyphla concept and in support of hedgehogs as the sister group to shrews. Molecular Phylogenetics and Evolution 25:200-209.

DouAdy, C. J., Y E. J. Douzery. 2003. Molecular estimation of eulipotyphlan divergence times and the evolution of "Insectivora". Molecular Phylogenetics and Evolution 28:285-296.

Escalante-Espinosa, T. 2003. Determinación de prioridades en las áreas de conservación para los mamíferos terrestres de México, empleando criterios biogeográficos. Anales del Instituto de Biología, Serie Zoología 74:211-237.

Esteva, M. E., F. A .Cervantes, S. Brant, y J. Cook. 2010. Molecular phylogeny of long-tailed shrews (genus Sorex) from México and Guatemala. Zootaxa 2615:47-65.

Goldman, E. A. 1951. Biological investigations in México. Smithsonian Miscellaneous Collections 115. Washington, EE.UU.

Graham, C. H., S. Ferrier, F. Huettman, C. Moritz, y A. T. Peterson. 2004. New developments in museum-based informatics and applications in biodiversity analysis. Trends in Ecology and Evolution 19:497-503.

Guevara, L., H. E. Ramírez-Chaves, y F. A. Cervantes. 2011. Leucismo en la musaraña de orejas cortas Cryptotis mexicana (Mammalia: Soricomorpha), endémica de México. Revista Mexicana de Biodiversidad 82:731-733.

Guevara, L., C. Lorenzo, S. Ortega-García, y V. Sánchez-Cordero. 2014a. Noteworthy records of an endemic shrew from México (Mammalia, Soricomorpha, Cryptotis griseoventris), with comments on taxonomy. Mammalia 78: 405-408.

Guevara, L., V. Sánchez-Cordero, L. León-Paniagua, y N. Woodman. 2014b. A new species of small-eared shrew (Mammalia, Eulipotyphla, Cryptotis) from the Lacandona rainforest, México. Journal of Mammalogy 95:739-753. 
Guevara, L., y F. A. Cervantes. 2014. Molecular systematics of small-eared shrews (Soricomorpha, Mammalia) within Cryptotis mexicanus species group from Mesoamérica. Acta Theriologica 59:233-242.

Hutterer, R. 2005. Order Soricomorpha. Pp. 220-311 in Mammal Species of the world: A taxonomic and geographic reference (Wilson, D. E., y D. M. Reeder, eds.). The John Hopkins University Press. Baltimore, EE.UU.

INEGI (Instituto Nacional de Estadística y Geografía). 2014. Uso del Suelo y Vegetación escala 1:250,000, Serie V, Departamento de Uso del Suelo, Dirección General de Geografía, Instituto Nacional de Estadística, Geografía e Informática, México. Sitio del INEGI en Internet: www.inegi.org.mx

iUCN (International Union for Conservation of Nature). 2013. IUCN Red List of Threatened Species. Version 2013.1.

iUCN (International Union for Conservation of Nature), y UNEP (United Nations Environment Programme). 2014. The World Database on Protected Areas (WDPA). UNEP-WCMC. Cambridge, United Kingdom.

iCZN (International Commission on Zoological Nomenclature). 1999. International code of zoological nomenclature. Cuarta edicion International Trust for Zoological Nomenclature. London, United Kingdom.

iCZN (International Commission on Zoological Nomenclature). 2006. Opinion 2164 (Case 3328). Didelphis Linnaeus, 1758 (Mammalia, Didelphidae): gender corrected to feminine, and Cryptotis Pomel, 1848 (Mammalia, Soricidae): gender fixed as masculine. Bulletin of Zoological Nomenclature 63:282-283.

JaCkson, C. R., Y M. P. Robertson. 2011. Predicting the potential distribution of an endangered cryptic subterranean mammal from few occurrence records. Journal for Nature Conservation 19:87-94.

Maldonado, J. E. 1999. Family Talpidae. Pp: 53-55 en Mamíferos del noroeste de México (Álvarez-Castañeda S. T., y J. L. Patton, eds.). Centro de Investigaciones Biológicas del Noroeste, S. C. La Paz, Baja California Sur, México.

Matson, J. O., Y T. J. McCarthy. 2005. A new subspecies of Verapaz shrew (Sorex veraepacis) from Guatemala. Pp. 63-70 en Advances in the Biology of Shrews II (Merritt, J. F., S. Churchfield, R. Hutterer, y B. I. Sheftel, eds.). Special Publication of the International Society of Shrew Biologist. New York, EE.UU.

Merritt, J. F. 2010. The biology of small mammals. The Johns Hopkins University Press. Baltimore, EE.UU.

Naranjo, E. J., y E. Espinoza. 2001. Los mamíferos de la reserva ecológica Huitepec, Chiapas, México. Revista Mexicana de Mastozoología 5:58-67.

Nikaido, M., Y. CaO, M. Harada, N. Okada, y M. Hasegawa. 2003. Mitochondrial phylogeny of hedgehogs and monophyly of Eulipotyphla. Molecular Phylogenetics and Evolution 28:276-284.

PacC (Programa de Acción ante el Cambio Climático en el Estado de Chiapas). 2011. Anexo técnico III Modelo de Deforestación del Estado de Chiapas. Secretaría de Medio Ambiente e Historia Natural. Disponible en: http://www2.inecc.gob.mx/sistemas/ peacc/descargas/paccc_chiapas.pdf. Consultado el 14 de mayo de 2013.

Patterson, B. D. 2002. On the continuing need for scientific collecting of mammals. Mastozoología Neotropical 9:253-262.

Pearson, R. G., C. J. Raxworthy, M. Nakamura, y A. T. Peterson. 2007. Predicting species distributions from small numbers of occurrence records: a test case using cryptic geckos in Madagascar. Journal of Biogeography 34:102-117. 
Peterson, A. T., V. Sánchez-Cordero, J. Soberón, J. Bartley, R. W. Buddemeier, y A. NavarroSigüEnZA. 2001. Effects of global climate change on geographic distributions of Mexican Cracidae. Ecological Modeling 144:21-30.

Peterson, A.T., J. Soberón, R. G. Pearson, R. P. Anderson, E. Martínez-Meyer, M. Nakamura, y M. B. Araújo. 2011. Ecological Niches and Geographic Distributions. Monographs in Population Biology. Princeton University Press. Princeton, EE.UU.

Phillips, S. J., R. P. Anderson, y R. E. SchapiRe. 2006. Maximum entropy modeling of species geographic distributions. Ecological Modelling 190:231-259.

Phillips, S. J., M. Dudík, J. Elith, C. H. Graham, A. Lehmann, A., J. Leathwick, y S. Ferrier. 2009. Sample selection bias and presence-only distribution models: implications for background and pseudo-absence data. Ecological Applications 19:181-197.

Ponce-Reyes, R., V. H. Reynoso-Rosales, J. E. M. Watson, J. VanDerWal, R. A. Fuller, R. L. Pressey, y H. P. Possingham. 2012. Vulnerability of cloud forest reserves in Mexico to climate change. Nature Climate Change 2:448-452.

Ramírez-Pulido, J., A. Castillo-Morales, A. Salame-Méndez, y A. Castro-Campillo. 2004. Características morfológicas y morfométricas de cinco especies de Cryptotis (Mammalia: Soricomorpha). Acta Zoológica Mexicana 20:9-37.

Ramírez-Pulido, J., A. Castillo-Morales, A. Salame-Méndez, A. Castro-Campillo, A., y N. González-Ruíz. 2005. Variación y distribución de Sorex (Mammalia: Soricomorpha) del centro de México. Revista de la Sociedad Mexicana de Historia Natural 3ra. Época 2:178-192.

Ramírez-Pulido, J., J. Arroyo-Cabrales, y N. González-Ruíz. 2008. Mamíferos. CD 1 en Catálogo taxonómico de especies de México, Vol. I: Conocimiento actual de la biodiversidad (Ocegueda, S., y J. Llorente-Bousquets, coord.). Comisión Nacional para el Conocimiento y Uso de la Biodiversidad. Ciudad de México, México.

Ramírez-Pulido, J., N. González-Ruiz, A. L. Gardner, y J. Arroyo-Cabrales. 2014. List of Recent Land Mammals of Mexico, 2014. Special Publications Museum of Texas Tech University 63:1-69.

Raxworthy, C. J., E. Martinez-Meyer, N. Horning, R. A. Nussbaum, G. E. Schneider, M. A. OrtegaHuerta, y A. T. Peterson. 2003. Predicting distributions of known and unknown reptile species in Madagascar. Nature 426:837-841.

Roca, A. L., G. Bar-Gal, K., E. EiziriK, K. M. Helgen, R. Maria, R., M. S. Springer, S. J. O'brien, y W. J. Murphy. 2004. Mesozoic origin for West Indian insectivores. Nature 429:649651.

Rocha, L. A., A. Aleixo, G. Allen, F. Almeda, C. C. Baldwin, M. V. Barclay, J. M. Bates, A. M. Bauer, F. Benzoni, C. M. Berns, M. L. Berumen, D. C. Blackburn, S. Blum, F. Bolaños, R. C. K. Bowie, R. Britz, R. M. Brown, C. D. Cadena, K. Carpenter, L. M. Ceríaco, P. Chakrabarty, G. Chaves, J. H. Choat, K. D. Clements, B. B. Collette, A. Collins, J. Coyne, J. Cracraft, T. Daniel, M. R. de Carvalho, K. de Queiroz, F. Di Dario, R. Drewes, J. P. Dumbacher, A. Engilis JR., M. V. Erdmann, W. Eschmeyer, C. R. Feldman, B. L. Fisher, J. FJeldså, P. W. Fritsch, J. Fuchs, A. Getahun, A. Gill, M. Gomon, T. Gosliner, G. R. Graves, C. E. Griswold, R. Guralnick, K. Hartel, K. M. Helgen, H. Ho, D. T. Iskandar, T. Imamoto, Z. JaAfar, H. F. James, D. Johnson, D. Kavanaugh, N. Knowlton, E. Lacey, H. K. Larson, P. Last, J. M. Leis, H. Lessios, J. Liebherr, M. Lowman, D. L. Mahler, V. Mamonekene, K. Matsuura, G. C. Mayer, H. Mays JR., J. McCosker, R. W. McDiarmid, J. McGuire, M. J. Miller, R. MooI, R. D. Mool, C. Moritz, P. Myers, M. W. Nachman, R. A. Nussbaum, D. Ó Foighil, L. R. Parenti, J. F. Parham, E. Paul, G. Paulay, J. Pérez-Emán, A. Pérez-Matus, S. Poe, J. Pogonoski, D. L. Rabosky, J. E. Randall, J. D. Reimer, D. R. Robertson, M.-O. Rödel, M. T. Rodrigues, P. 
Roopnarine, L. Rüber, M. J. Ryan, F. Sheldon, G. Shinohara, A. Short, W. B. Simison, W. F. Smith-Vaniz, V. G. Springer, M. Stiassny, J. G. Tello, C. W. Thompson, T. Trnski, P. Tucker, T. Valqui, M. Vecchione, E. Verheyen, P. C. Wainwright, T. A. Wheeler, W. T. White, K. Will, J. T. Williams, G. Williams, E. O. Wilson, K. Winker, R. Winterbottom, y C. C. Witt. 2014. Specimen collection: an essential tool. Science 344:814-815.

Sánchez-Cordero, V., A. T. Peterson, y P. Escalante-Pliego. 2001. El modelado de la distribución de especies y la conservación de la diversidad biológica. Pp. 359-379 en Enfoques contemporáneos para el estudio de la biodiversidad (Hernández, H. M., A. N. García-Aldrete, F. Álvarez, y M. Ulloa, eds.). Ediciones Científicas Universitarias Fondo de Cultura Económica, Academia Mexicana de Ciencias, A.C., Instituto de Biología, Universidad Nacional Autónoma de México. Ciudad de México, México.

Sánchez-Cordero, V., P. Illoldi-Rangel, M. Linaje, S. Sarkar, y A. T. Peterson. 2005. Deforestation and extant distributions of Mexican endemic mammals. Biological Conservation 126:465-473.

Sánchez-Cordero, V., P. Illoldi-Rangel, M. Linaje, T. Escalante, F. Figueroa, y S. Sarkar. 2009. Deforestation and biodiversity conservation in Mexico. Pp. 279-298 en Endangered Species: New Research (Columbus, A., y L. Kuznetsov, eds.). Nova Science Publishers. New Haven, EE.UU.

Schloss, C. A., T. A. Nuñez, y J. J. LaWler. 2012. Dispersal will limit ability of mammals to track climate change in the Western Hemisphere. Proceedings of the National Academy of Sciences 109:8606-8611.

Semarnat (Secretaría de Medio Ambiente y Recursos Naturales). 2010. Norma Oficial Mexicana NOM-059-SEMARNAT-2010, Protección ambiental-Especies nativas de México de flora y fauna silvestres-Categorías de riesgo y especificaciones para la inclusión, exclusión o cambio-Lista de especies en riesgo. Diario Oficial de la Federación. Ciudad de México, México.

Simpson, G. G. 1945. The principles of classification and a classification of mammals. Bulletin of the American Museum of Natural History 85:1-350.

Soberón, J., y A. T. Peterson. 2005. Interpretation of models of fundamental ecological niches and species' distributional areas. Biodiversity Informatics 2:1-10.

Stanhope, M. J., V. G. Waddell, O. Madsen, W. De Jong, S. B. Hedges, G. C. Cleven, K. Diana, y M. S. Springer. 1998. Molecular evidence for multiple origins of Insectivora and for a new order of endemic African insectivore mammals. Proceedings of the National Academy of Sciences 95:9967-9972.

STATSOFT INC. 2005. STATISTICA (data analysis software system) version 7.1. Disponible en: http://www.statsoft.com/. Tulsa, EE.UU.

Symonds, M. R. 2005. Phylogeny and life histories of the 'Insectivora': controversies and consequences. Biological Reviews 80:93-128.

Trejo, I., E. Martínez-Meyer, E. Calixto-Pérez, S. Sánchez-Colón, R. Vázquez de la Torre, y L. Vituers-Ruiz. 2011. Analysis of the effects of climate change on plant communities and mammals in México. Atmósfera 24:1-14.

Vázquez, L. B., C. G. Bustamante-Rodríguez, y D. G. Bahena-Arce. 2009. Area selection for conservation of Mexican mammals. Animal Biodiversity and Conservation 32.1:29-39.

Yates, T. L., Y J. Salazar-Bravo. 2005. A revision of Scapanus latimanus, with the revalidation of a species of Mexican mole. Pp. 479-496 en Contribuciones mastozoológicas en homenaje a Bernardo Villa (Sánchez-Cordero, V., y R. Medellín, eds.). Instituto de Biología e Instituto de Ecología, UNAM. Ciudad de México, México. 
Warren, D. L., R. E. Glor, y M. Turelli. 2010. ENMTools: a toolbox for comparative studies of environmental niche models. Ecography 33:607-611.

WarRen, D. L., Y S. N. Selfert. 2011. Ecological niche modeling in Maxent: the importance of model complexity and the performance of model selection criteria. Ecological Applications 21:335-342.

Wilson, D. E., Y D. M. Reeder. 2011. Class Mammalia Linnaeus, 1758. In Animal biodiversity: An outline of higher-level classification and survey of taxonomic richness. Zootaxa 3148:56-60.

Woodman, N., y D. A. Croft. 2005. Fossil shrews from Honduras and their significance for late glacial evolution in body size (Mammalia: Soricidae: Cryptotis). Fieldiana Geology 51:1-30.

Woodman, N. 2010. Two new species of shrews (Soricidae) from the western highlands of Guatemala. Journal of Mammalogy 91:566-579.

Woodman, N. 2012. Taxonomic status and relationships of Sorex obscurus parvidens Jackson, 1921, from California. Journal of Mammalogy 93:826-838.

Woodman, N., J. O. Matson, T. J. McCarthy, R. P. Eckerlin, W. Bulmer, y N. Ordóñez-Garza. 2012. Distributional records of shrews (Mammalia, Soricomorpha, Soricidae) from Northern Central America with the first record of Sorex from Honduras. Annals of Carnegie Museum 80:207-237.

Summited: July 2, 2014

Review: August 22, 2014

Accepted: October 20, 2014

Associated editor: Consuelo Lorenzo 


\section{Apéndice 1}

Distribución actual de 2 especies de topos de los géneros Scalopus y Scapanus y 25 de musarañas de los géneros Cryptotis, Megasorex, Notiosorex y Sorex dentro de México. De izquierda a derecha se muestra el nombre científico, la distribución actual (azul) con las localidades registradas para México (puntos en rojo) y la ubicación de las localidades con las que se generaron los modelos (cruces en rojo) en el espacio ambiental: temperatura promedio anual (TMA, eje $\mathrm{x}$ ) y precipitación anual (PA, eje y). También se muestran los valores para ambas variables para México (puntos negros) y para el área de calibración del modelo (puntos verdes). Al final, se presentan las localidades para las especies para las cuales no se generaron modelos de nicho ecológico.

Cryptotis alticola
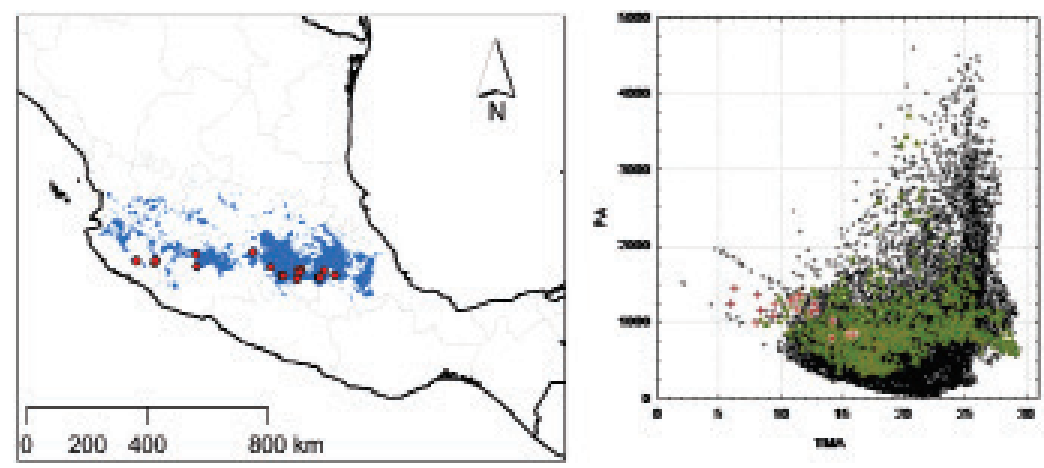

C. goldmani
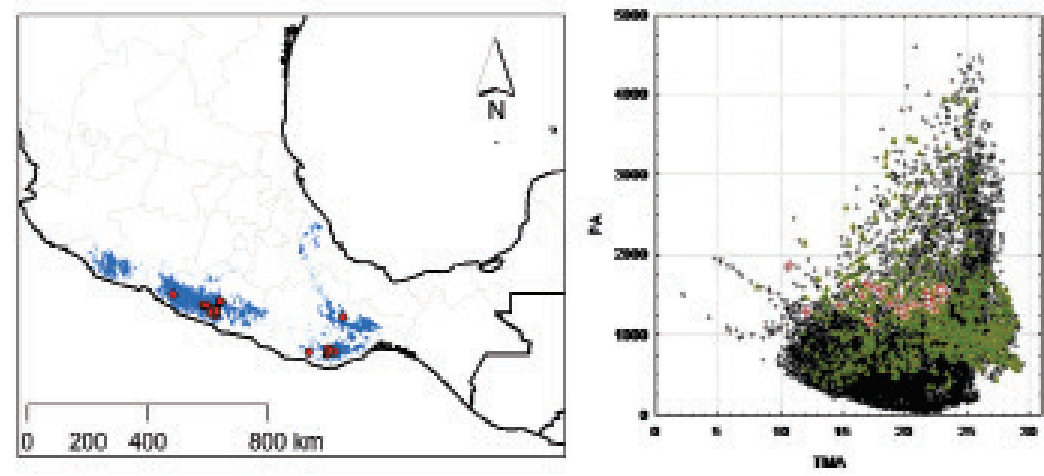

C. goodwini
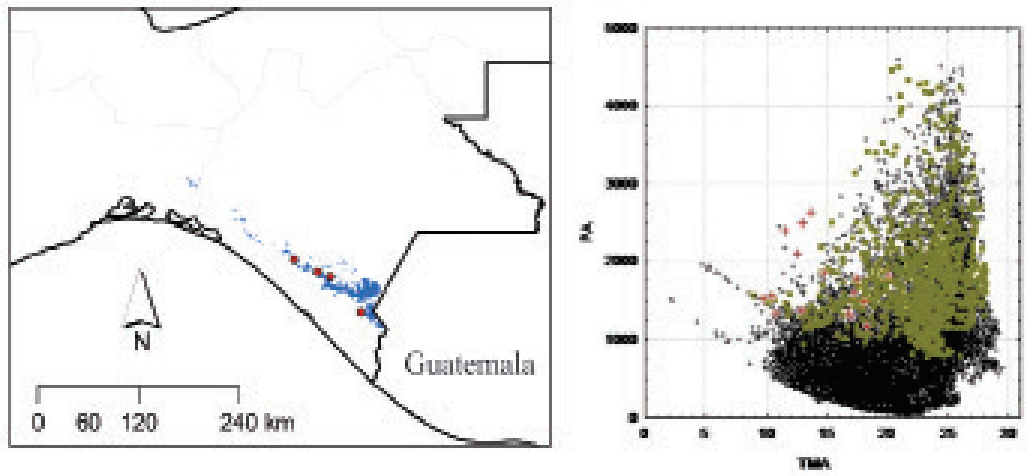

\section{C. griseoventris}
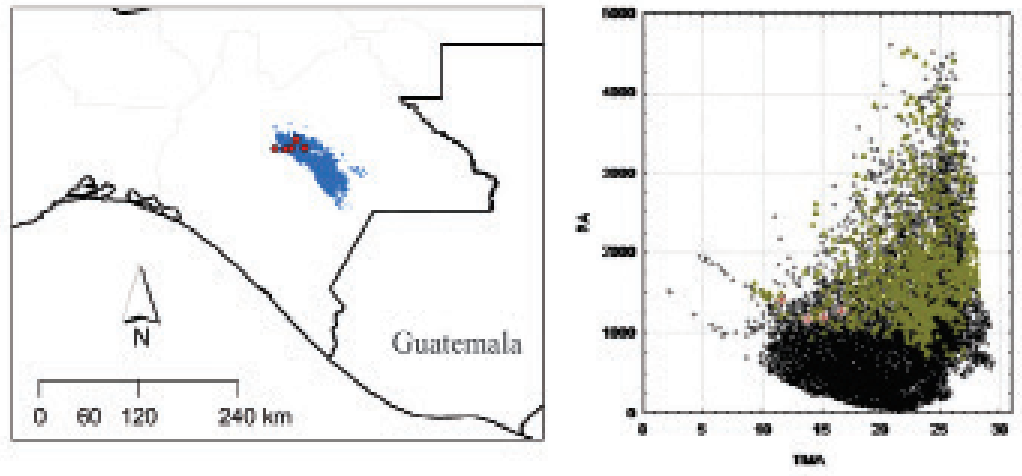
C. magnus
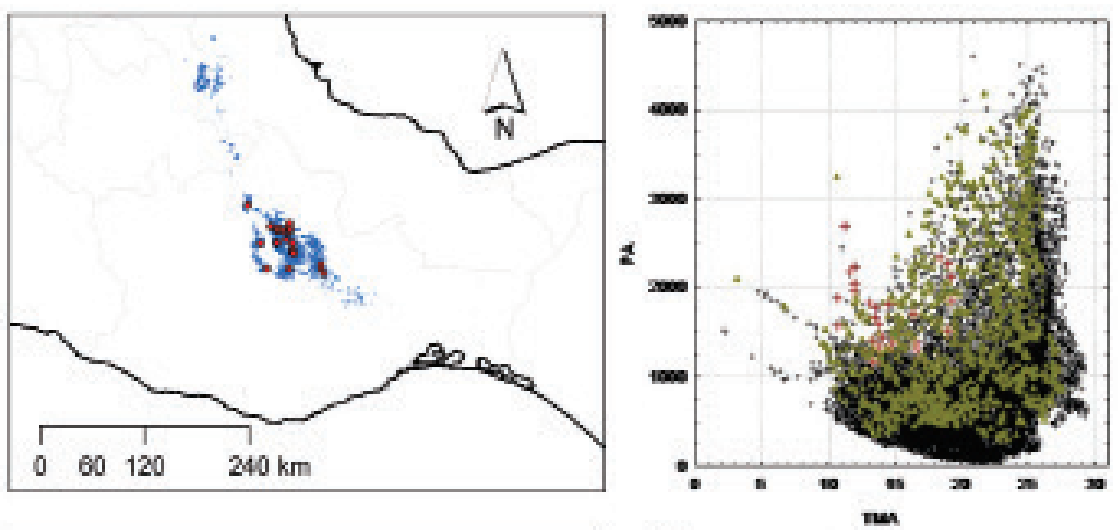

C. mayensis
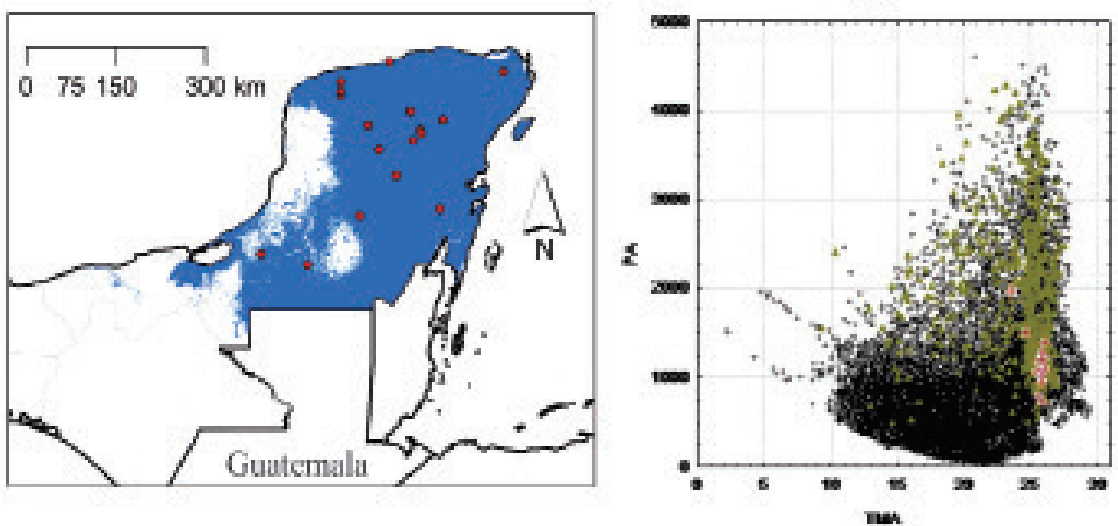

C. merriami
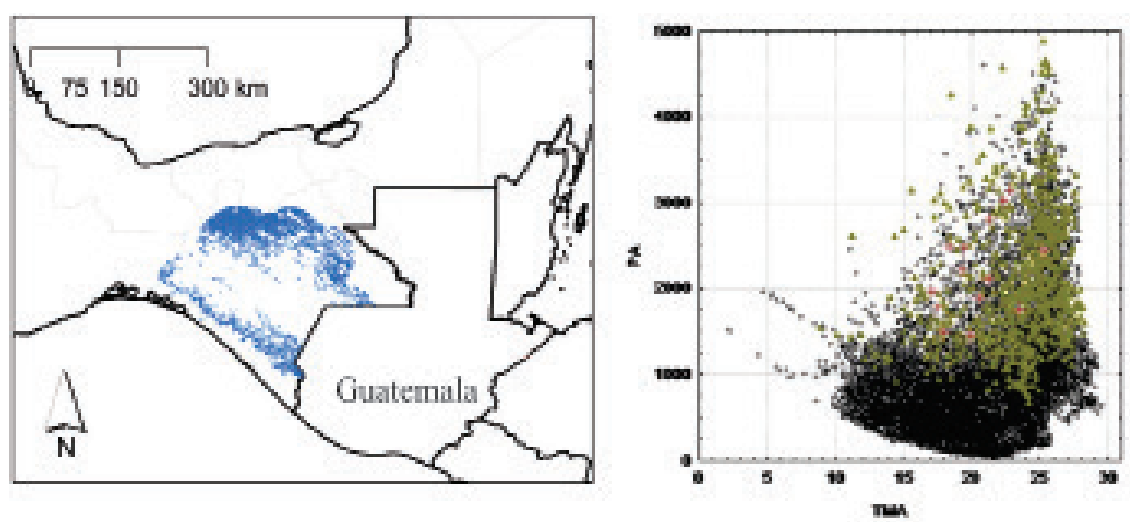

C. mexicanus
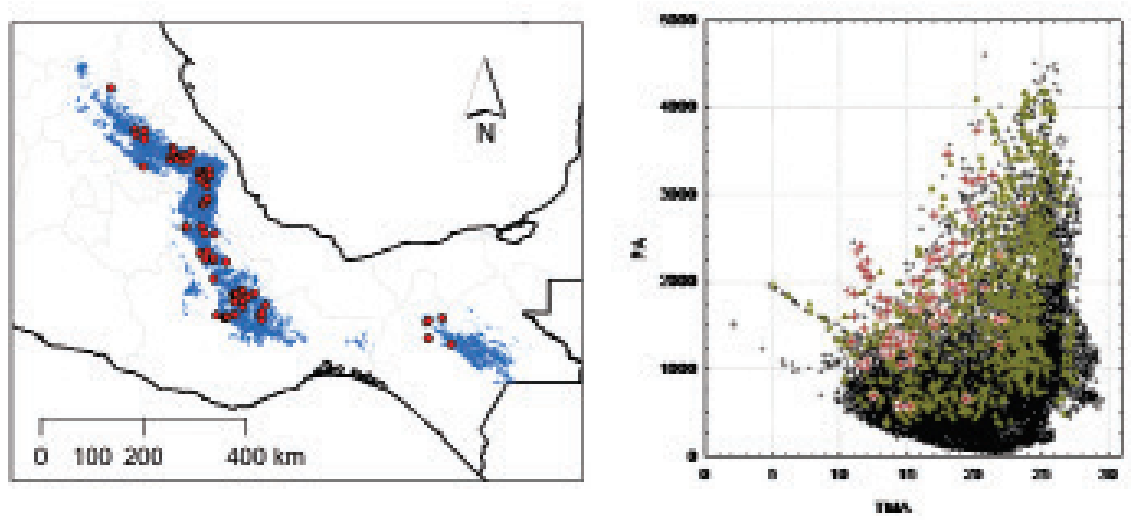
C. obscurus
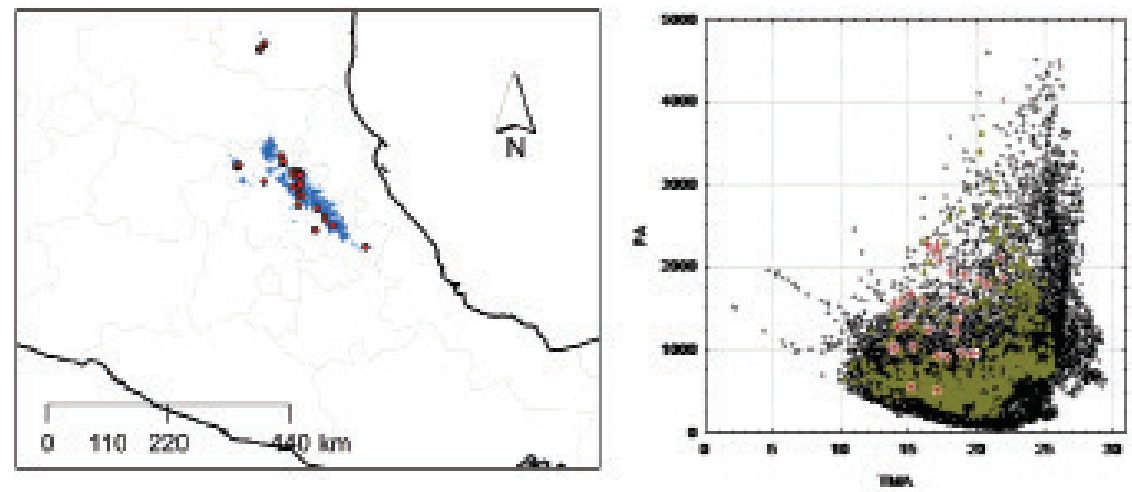

C. parvus
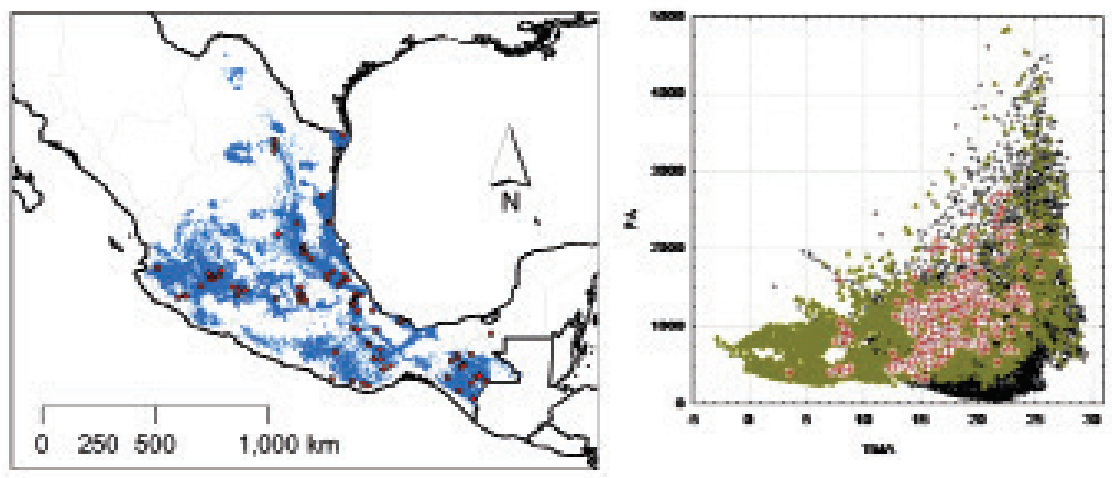

C. peregrimus
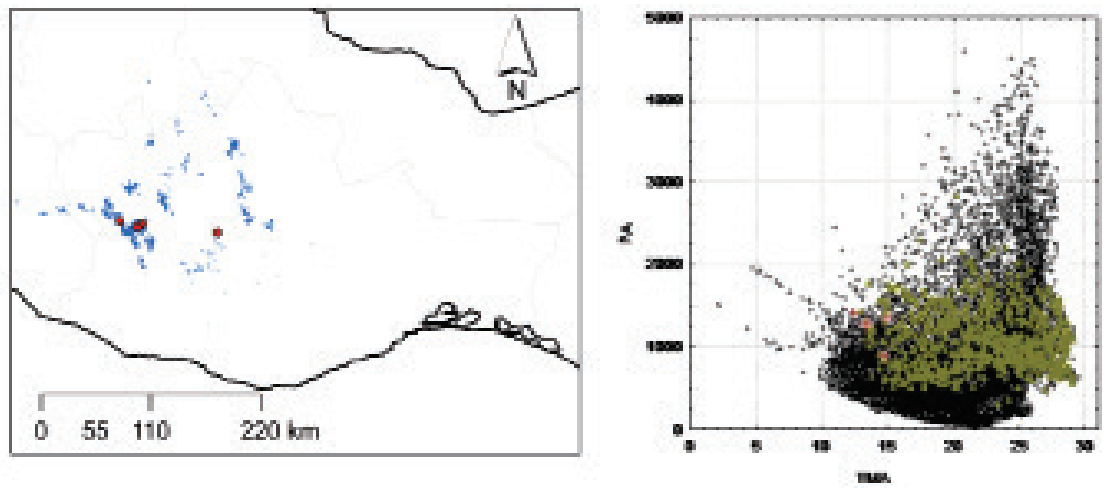

\section{C. phillipsii}
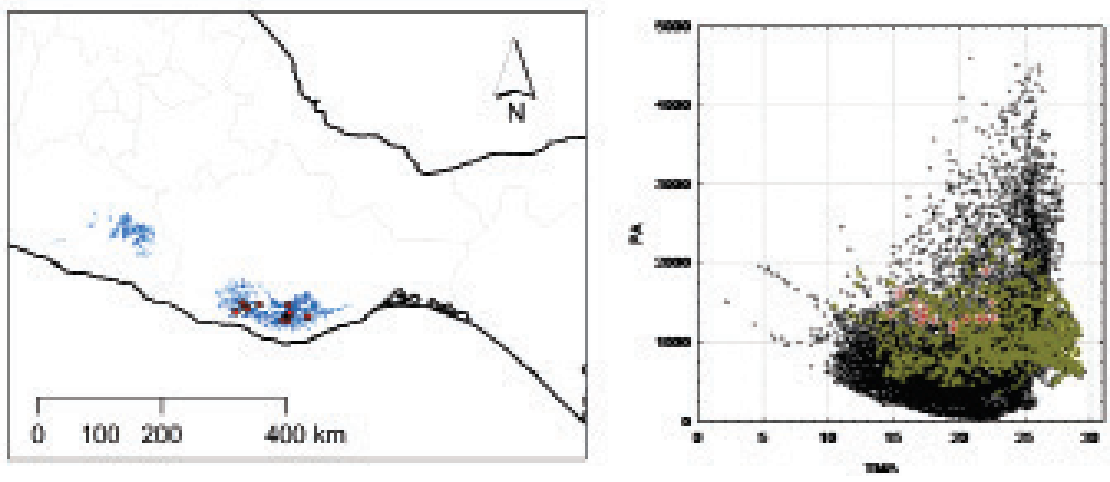
Megasorex gigas
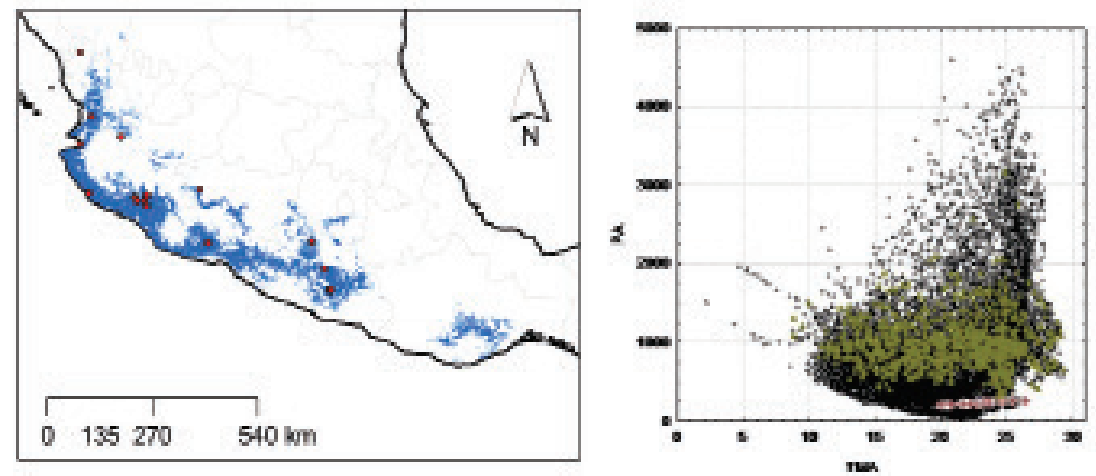

Notiosorex crawfordi
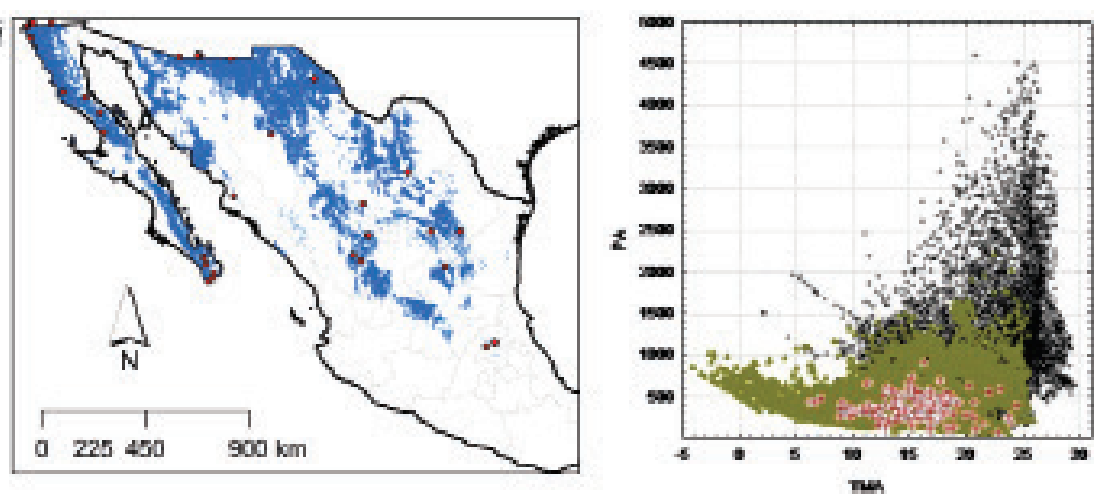

N. evotis
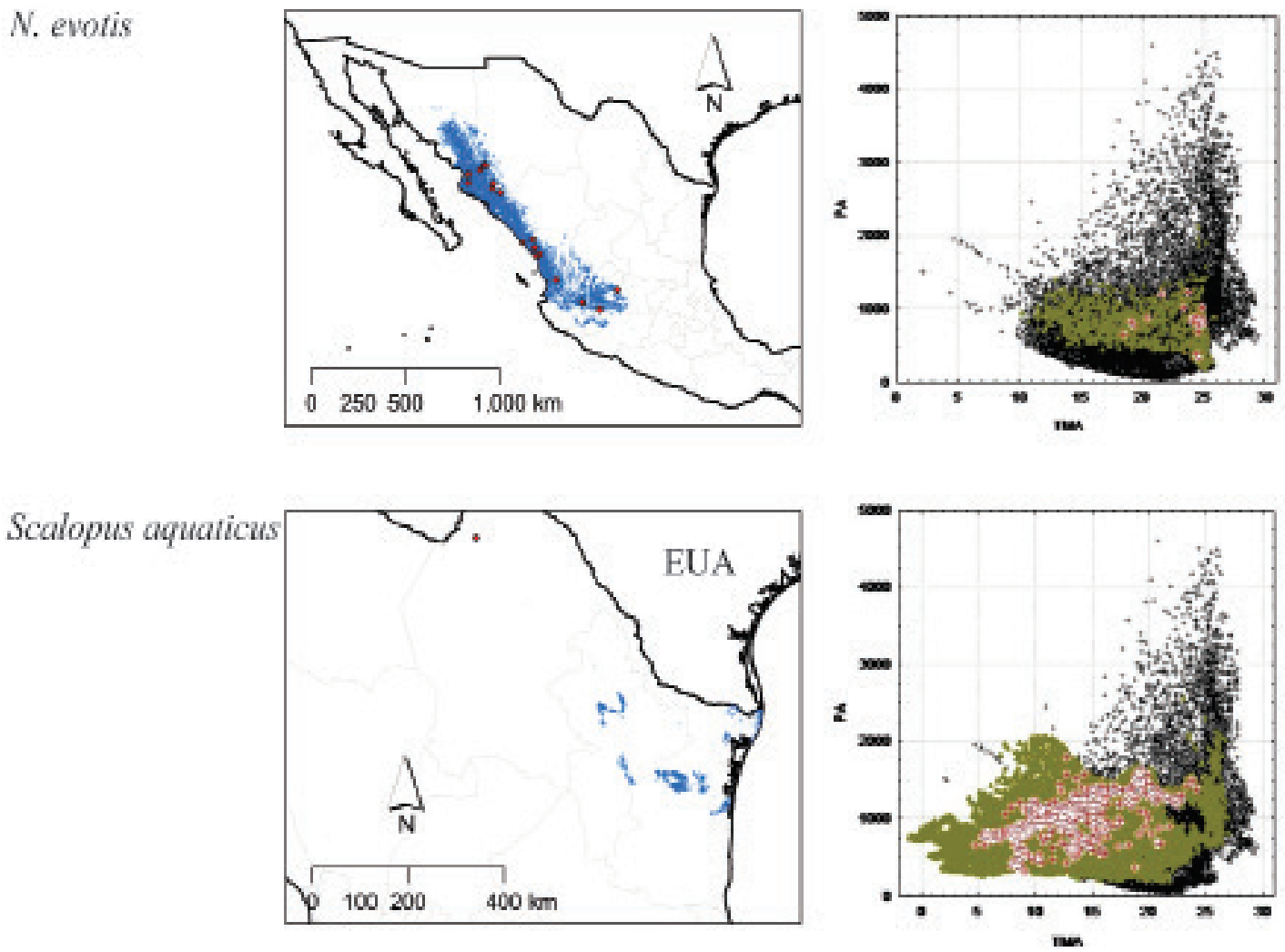
Sorex arizonae
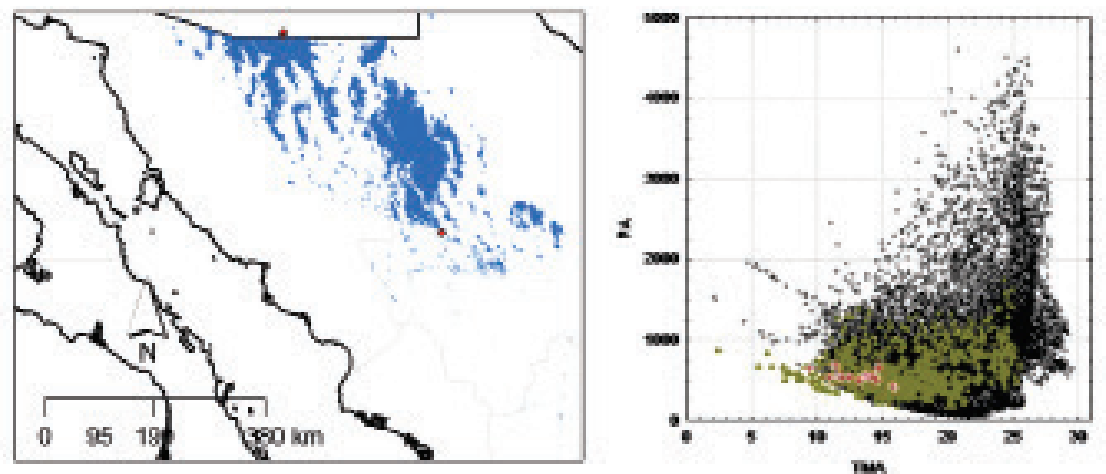

\section{S. ixtlanensis}
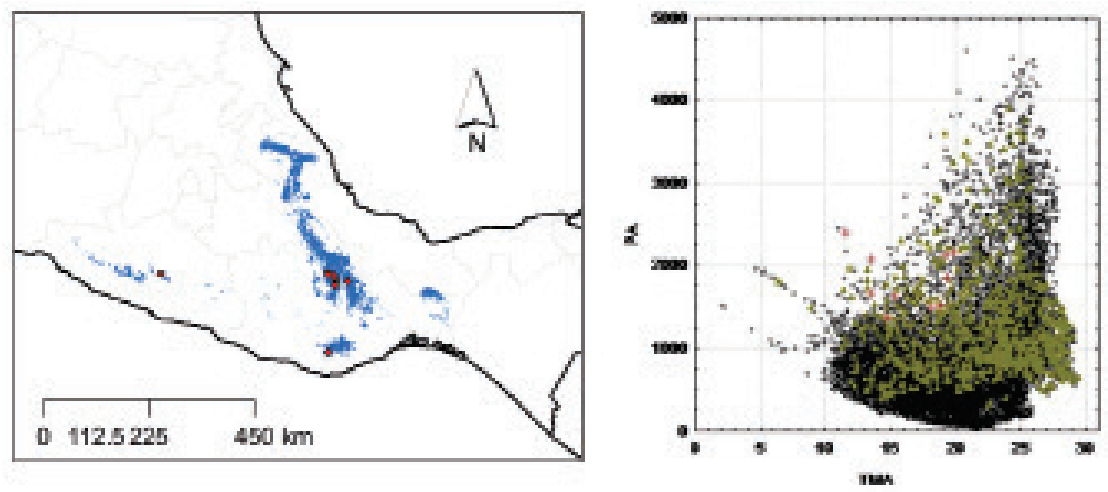

S. milleri
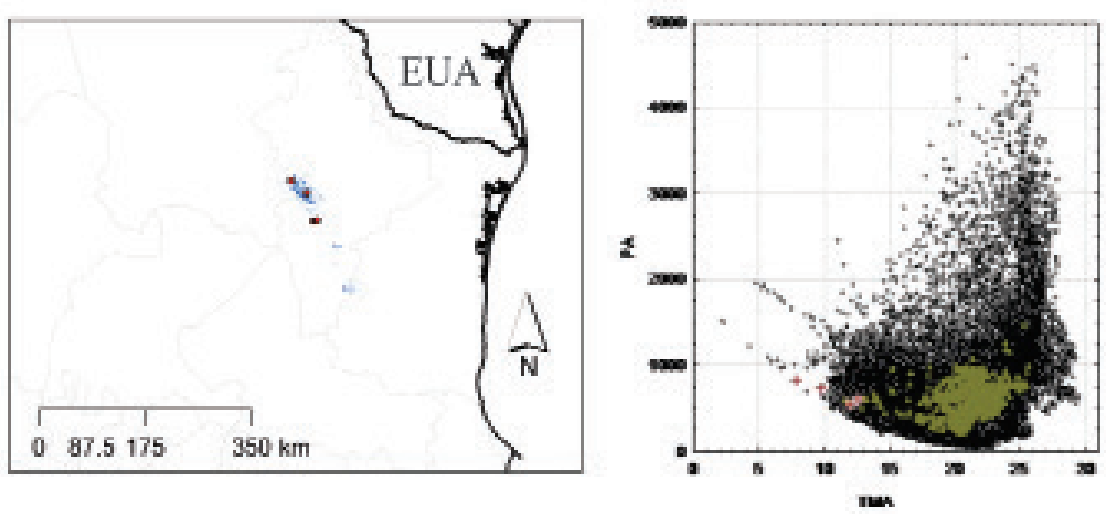

S, monticola
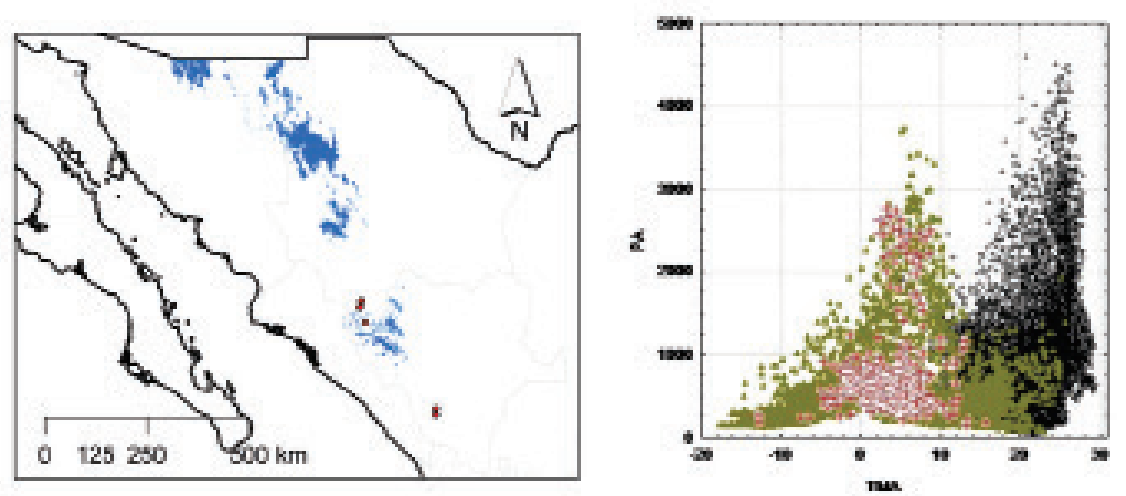
S. oreopolus
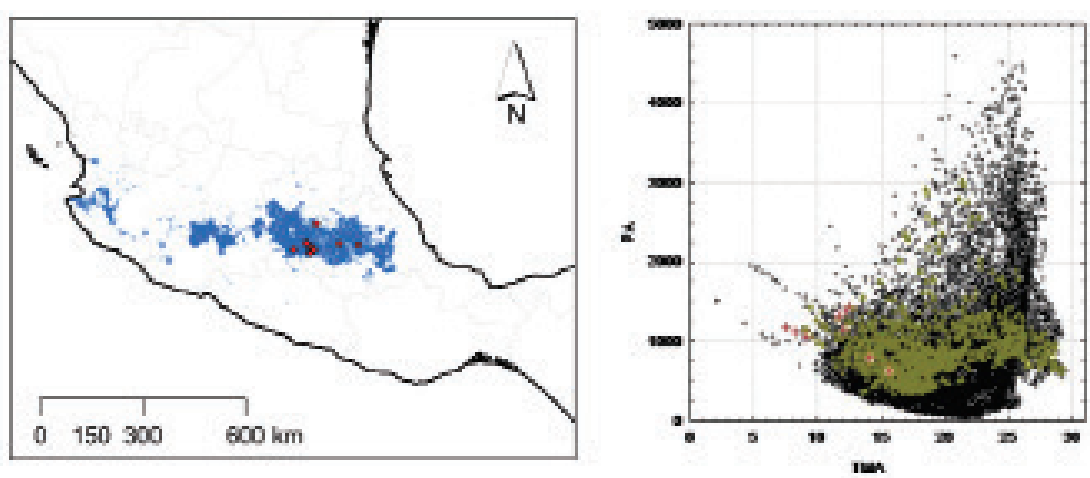

S. ornatus
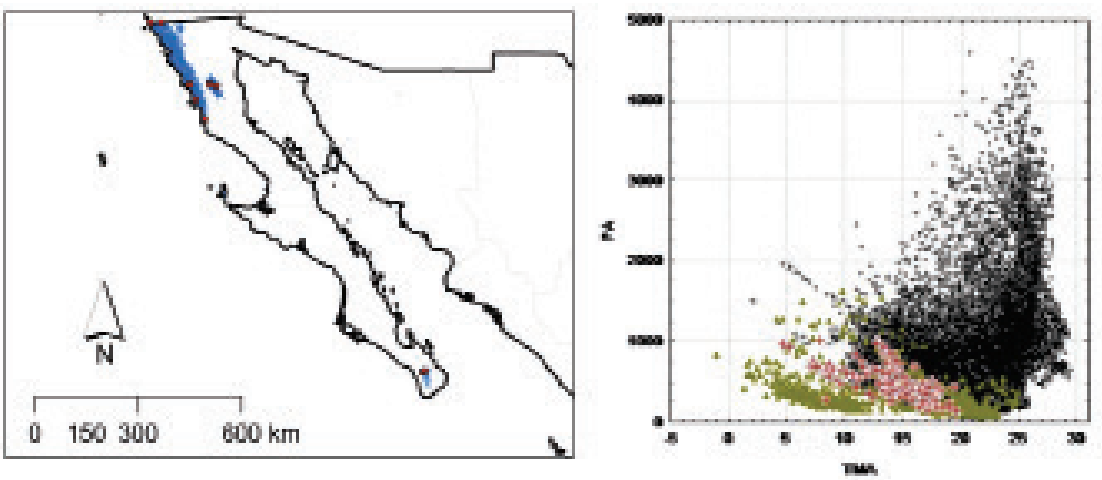

S. saussurel
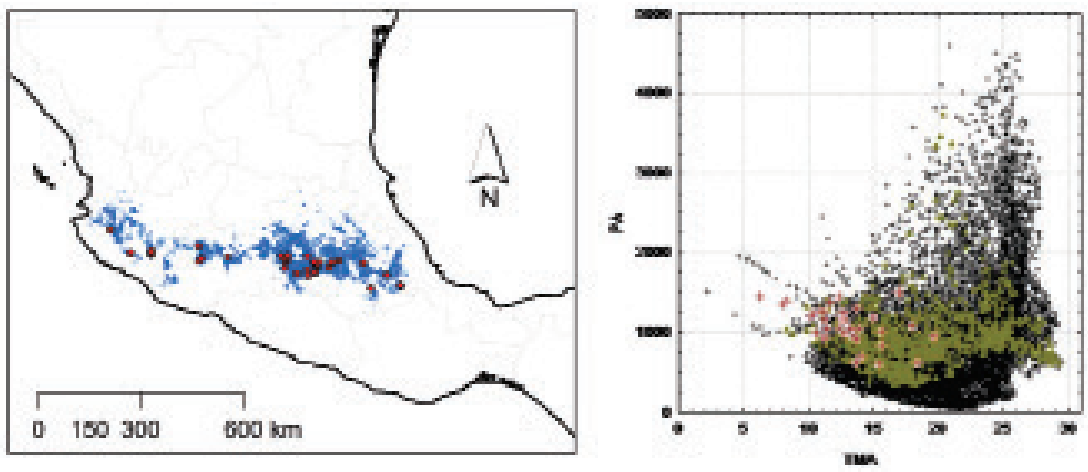

$S$, ventralis
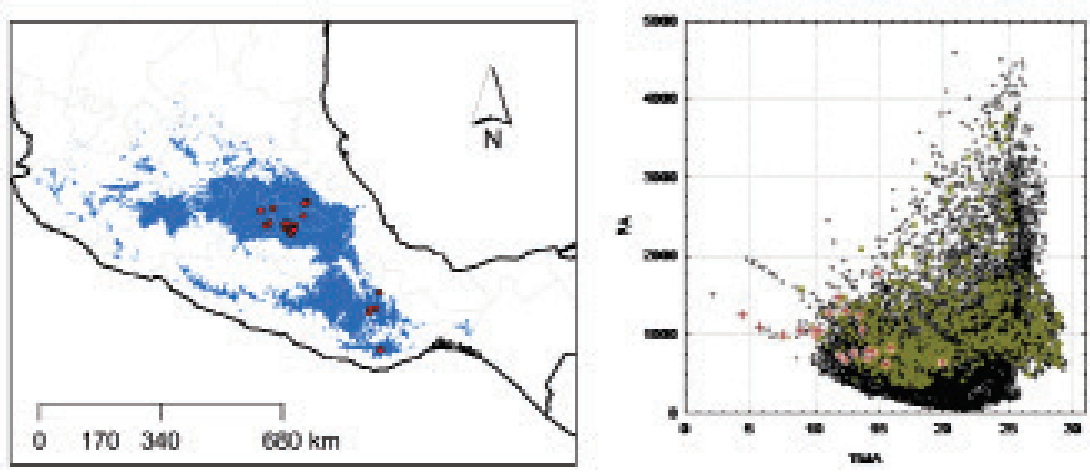
S. salvini
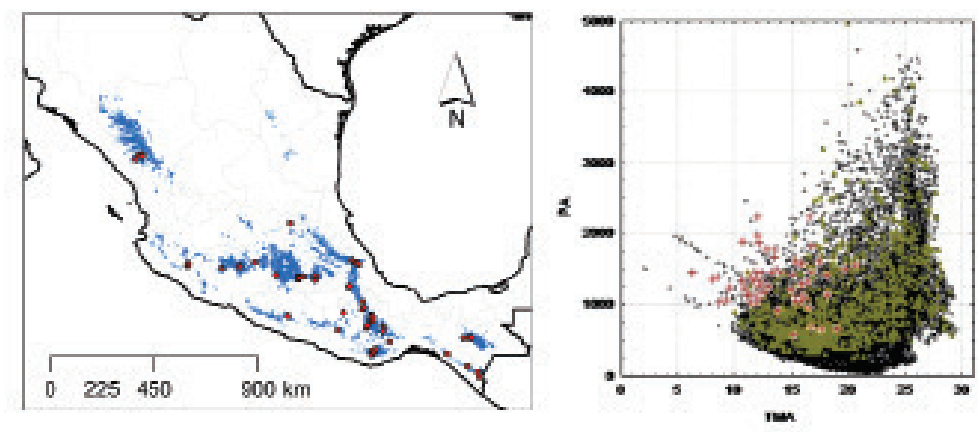

S. veraepacis
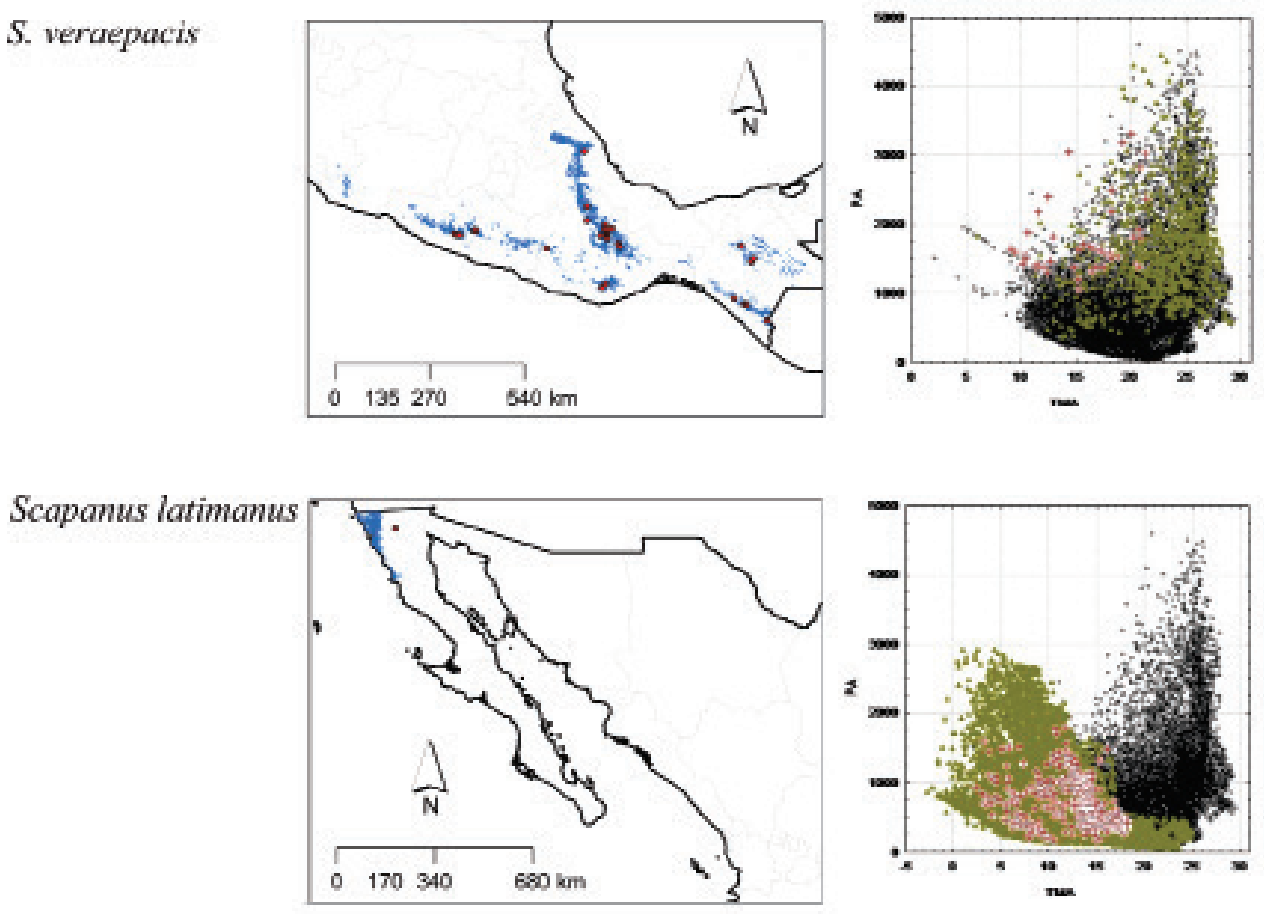

Especies sin modelo de nicho ecológico (ver Materiales y métodos)
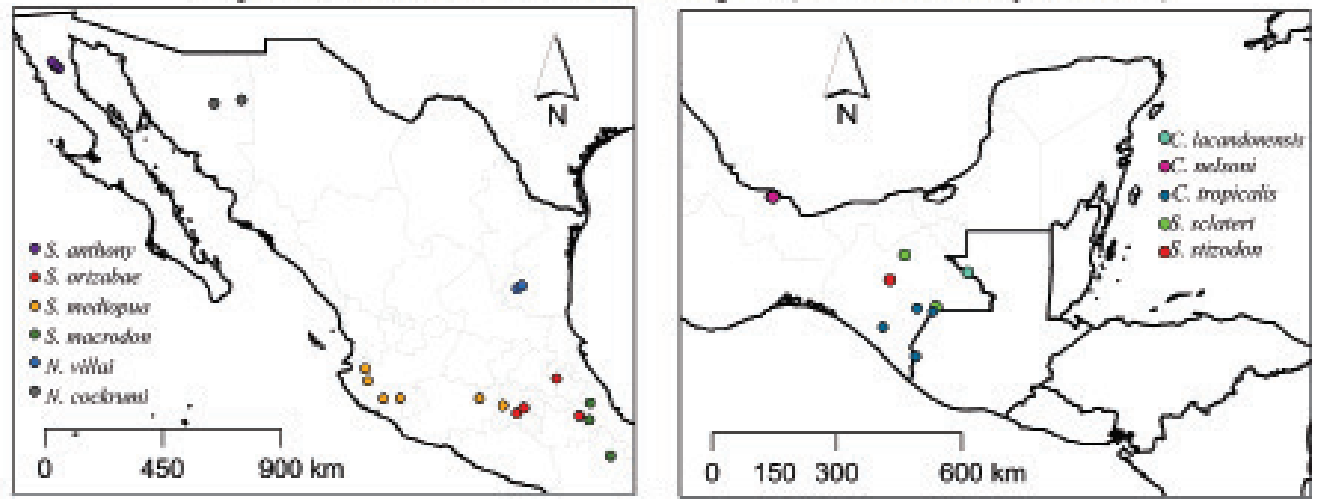